\title{
Active and Passive Helicopter Noise Reduction Using the AVINOR/HELINOIR Code Suite
}

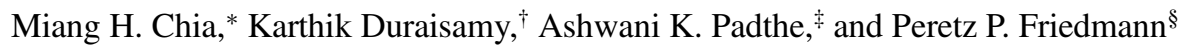 \\ University of Michigan, Ann Arbor, Michigan 48109-2140
}

DOI: $\underline{10.2514 / 1 . C 034519}$

\begin{abstract}
A suite of computational tools capable of predicting in-plane low-frequency rotorcraft noise and its control using blade-tip geometry modifications is developed. The combined code, consisting of AVINOR, a comprehensive rotorcraft analysis code, and an acoustic code called HELINOIR, is first validated against wind-tunnel tests and subsequently verified by comparing with computational results. Three rotor configurations resembling the Messerschmitt-Bölkow-Blohm BO105 with a tip sweep, dihedral, and anhedral were simulated for level flight at a moderate advance ratio. The impact of passive blade geometry modification on in-plane noise and vibration is studied and compared to the in-plane noise reduction obtained using a single $20 \%$ chord active plain trailing-edge flap with a feedback microphone on the left boom. Active control, which is implemented using an adaptive higher harmonic control algorithm, reduces in-plane low-frequency sound pressure levels below the horizon by up to $6 \mathrm{~dB}$, but there is an increase in vibratory loads. The tip dihedral reduces low-frequency sound pressure level by up to $2 \mathrm{~dB}$ without a vibratory load penalty, but there is an increase in the midfrequency sound pressure levels. The tip sweep and tip anhedral increase in-plane low-frequency sound pressure level below the horizon. There is a general tradeoff associated with in-plane low-frequency sound pressure level reduction, vibration performance, and midfrequency sound pressure level.
\end{abstract}

\begin{tabular}{|c|c|c|}
\hline$[C]$ & $=$ & damping matrix \\
\hline$C_{d f}$ & $=$ & fuselage drag coefficient \\
\hline$C_{W}$ & $=$ & helicopter weight coefficient \\
\hline$C_{T}$ & $=$ & thrust coefficient \\
\hline$c$ & $=$ & rotor blade chord \\
\hline$e$ & $=$ & blade root offset from center of rotation \\
\hline $\boldsymbol{F}$ & $=$ & load vector in the equation of motion \\
\hline$J$ & $=$ & quadratic cost function \\
\hline$[K]$ & $=$ & stiffness matrix \\
\hline$L_{b}$ & $=$ & nondimensional blade length \\
\hline$[M]$ & $=$ & mass matrix \\
\hline$M_{b}$ & $=$ & mass of one blade \\
\hline$N_{b}$ & $=$ & number of rotor blades \\
\hline$p$ & $=$ & acoustic pressure \\
\hline$Q$ & $=$ & weighting matrix for plant output \\
\hline$\widetilde{R}$ & $=$ & rotor blade radius \\
\hline $\boldsymbol{R}$ & $=$ & weighting on the control input \\
\hline$T$ & & $\begin{array}{l}\text { sensitivity matrix relating control input to the } \\
\text { plant output }\end{array}$ \\
\hline$u$ & $=$ & control input vector \\
\hline $\boldsymbol{W}$ & $=$ & matrix relating plant response to disturbance \\
\hline$w$ & $=$ & $\begin{array}{l}\text { disturbance vector representing the helicopter } \\
\text { operating condition }\end{array}$ \\
\hline$X_{A}$ & $=$ & $\begin{array}{l}\text { offset between the aerodynamic center and the } \\
\text { elastic axis }\end{array}$ \\
\hline
\end{tabular}

Presented as Paper 2017-1179 at the 55th AIAA Aerospace Sciences Meeting, Grapevine, TX, 09-13 January 2017; received 17 April 2017; revision received 10 July 2017; accepted for publication 16 July 2017; published online 7 September 2017. Copyright (C) 2017 by the American Institute of Aeronautics and Astronautics, Inc. All rights reserved. All requests for copying and permission to reprint should be submitted to CCC at www.copyright.com; employ the ISSN 0021-8669 (print) or 1533-3868 (online) to initiate your request. See also AIAA Rights and Permissions www.aiaa.org/randp

*Ph.D. Candidate, Department of Aerospace Engineering. Student Member AIAA.

${ }^{\dagger}$ Assistant Professor, Department of Aerospace Engineering. Member AIAA.

${ }^{*}$ Research Investigator, Department of Aerospace Engineering. Member AIAA.

${ }^{\S}$ François-Xavier Bagnoud Professor of Aerospace Engineering, Department of Aerospace Engineering. Fellow AIAA.

$\begin{array}{lll}X_{I b} & =\begin{array}{l}\text { offset of the blade cross-sectional center of mass } \\ \text { from the elastic axis }\end{array} \\ X_{F A}, Z_{F A} & =\begin{array}{l}\text { longitudinal and vertical offsets between rotor } \\ \text { hub and helicopter aerodynamic center }\end{array} \\ X_{F C}, Z_{F C} & =\begin{array}{l}\text { longitudinal and vertical offsets between rotor } \\ \text { hub and helicopter center of gravity }\end{array} \\ \boldsymbol{x} & =\text { observer location } \\ \boldsymbol{y} & =\text { state vector for the coupled aeroelastic model } \\ \boldsymbol{z} & =\text { plant output vector } \\ \alpha_{D} & =\text { descent angle } \\ \alpha_{R} & =\text { blade precone angle } \\ \beta_{p} & =\text { lock number } \\ \gamma & =\text { surface area of panel } \\ \Delta S & =\text { collective pitch } \\ \theta_{0} & =\text { tail rotor pitch angle } \\ \theta_{0 t} & =\text { cyclic pitch components } \\ \theta_{1 c}, \theta_{1 s} & =\text { blade pretwist distribution } \\ \theta_{t w} & =\text { advance ratio } \\ \mu & =\text { rotor solidity } \\ \sigma & =\text { lateral roll angle } \\ \phi_{R} & =\text { Azimuth angle } \\ \psi & =\text { rotor angular speed } \\ \Omega & =\text { blade flap, lag, and torsional natural frequencies } \\ \omega_{F}, \omega_{L}, \omega_{T} & \end{array}$

Subscripts

$\begin{array}{lll}i, j & = & \text { variable number } \\ L & & \text { loading noise } \\ \text { ret } & = & \text { retarded time } \\ T & & =\end{array}$

\section{Introduction}

$\mathbf{R}$ OTOR noise can be broadly classified based on its directivity and source. In-plane noise is the noise in the plane of the rotor and is composed of three main components, namely thickness, in-plane loading, and high-speed impulsive (HSI) noise. Out-ofplane noise is composed of two main components, blade-vortex interaction (BVI) and out-of-plane loading noise. The dominance of each component varies with flight condition. The out-of-plane BVI noise, which is dominant in a low-speed descending flight, can severely limit a civilian helicopter's community acceptance. In-plane, low-frequency rotorcraft noise is of particular concern from a military 
operation standpoint as it tends to propagate for long distances without significant attenuation, adversely affecting the aural detection range of a rotorcraft and hence its operational survivability. Helicopter noise suppression has been studied using passive means based on rotor blade geometry design modifications, mainly in the outboard $10 \%$ of the blade span, as well as active control methods.

Typical passive control methods consist of blade-tip planform modifications such as sweep, anhedral, dihedral, etc. For BVI noise, blade-tip shapes that produce vortex diffusion (e.g., Ogee tip) have been shown to be effective [1]. Blade sweep is also effective in reducing BVI noise by avoiding or delaying parallel interactions between the blade and the vortices [2]. For in-plane noise, thinning and tapering the tip of the rotor blade reduces the thickness noise contribution [3]. At high rotor tip speed, sweeping the blade reduces the effects of compressibility, effectively delaying delocalization and the onset of HSI noise [4]. However, the contribution of blade sweep at moderate tip speed has not been studied in detail. A rotor resembling that used in the British Experimental Rotor Program with a sweep was tested in the German-Dutch Wind Tunnel (DNW), and the measured in-plane noise spectra showed only marginal difference from the baseline unswept blade [5]. It was also shown in [6] that the sweep angle is not an important parameter, as compared to the taper ratio, because the sweep angle affects the quadrupole noise, which is not dominant at moderate tip speed. On the other hand, it was shown in [7] that the sweep angle introduces a phase shift effect between spanwise distributed source and sink couples, resulting in in-plane noise reduction. A tip anhedral, which is typically used to improve the hover figure of merit, and a tip dihedral, which is used for better forward flight performance, have also been studied for noisereduction application [8]. Based on wind-tunnel tests performed at the DNW, both the tip anhedral and dihedral were capable of BVI noise reduction. This was attributed to the tip anhedral/dihedral increasing the BVI miss distance $[2,9,10]$. However, there is only sparse literature available on the effects of tip anhedral/dihedral on in-plane noise.

Despite the valuable insights gained, these studies had limitations because some of them were purely computational fluid dynamics (CFD)-based aerodynamic studies, whereas the rest used simplistic structural dynamic models [8]. This is particularly questionable for swept and anhedral tips because they introduce strong bending torsion coupling, influencing blade vibrations and aeroelastic stability. Furthermore, in these studies, the adverse effects of noise reduction on the rotor hub vibratory loads or stability have not been explored. Studying the interaction between noise, vibrations, and stability is particularly critical for hingeless rotors. The effect of blade geometry modifications, such as sweep on the aeroelastic stability of hingeless rotors in hover and forward flight, was examined in detail in the research conducted by Celi and Friedmann $[11,12]$. For accurate prediction of helicopter noise and the effects of its suppression on the vibratory loads, it is essential to account for blade deflections and rotor trim using a high-fidelity rotor aeroelastic model coupled with an aeroacoustic model.

Several active control means such as higher harmonic control (HHC), pitch link actuated individual blade control, and on-blade control (OBC) implemented through a trailing-edge flap or a microflap have been studied for noise control [13]. These techniques modify the blade airloads to influence the BVI interactions for BVI noise reduction [3] or to generate an "antinoise" signal for in-plane noise reduction [14]. However, implementation of active control on a production helicopter has an associated cost that needs to be justified by sufficient benefits. Therefore, it is important to compare the noisereduction performance of the active approaches to other techniques such as passive blade geometry modification.

An accurate computational study of active/passive noise control and its influence on the hub vibrations requires a high-fidelity aeroelastic/aeroacoustic code that is also computationally efficient. The HELINOIR code combined with the Active Vibration and Noise Reduction (AVINOR) comprehensive rotorcraft analysis code [15] is well suited for such a study. The AVINOR code coupled with HELINOIR has been validated for out-of-plane BVI and in-plane noise prediction and verified for active in-plane noise control using an active flap [16]. The AVINOR/HELINOIR code suite is described with additional details in the current study to examine passive noise reduction using modified blade-tip planforms. The primary goal of this paper is to describe the Helicopter Noise Reduction (HELINOIR) $[17,18]$ aeroacoustic code and use it for both active and passive noise reduction. The impact of the noise suppression techniques on the vibratory loads is also examined. The specific objectives of the paper are as follows.

1) Provide a detailed description of the HELINOIR code.

2) Describe the combined AVINOR/HELINOIR aeroelasticaeroacoustic computational framework, including the tip planform and geometry modifications.

3) Use the aeroelastic-aeroacoustic framework for passive noise control while accounting for the potential vibration impact.

4) Compare performance of the passive methods to that of active on-blade control methods.

\section{Description of the Acoustic Methodology and HELINOIR Code}

The aeroacoustic computations are based on the solution of the Ffowcs-Williams and Hawkings [19] equations, using the Farassat 1 A formulation $[20,21]$. In this work, monopole and dipole sources of noise are considered, corresponding to the thickness and loading noise, respectively. These noise sources can be computed using the blade aeroelastic response and aerodynamic loading data from AVINOR directly, without the need for interpolation or approximation. Quadrupole or volume noise sources that arise due to phenomena such as shockwaves and separated flows are neglected. For the problem treated in this study, it is reasonable to assume that quadrupole noise sources are relatively unimportant when considering loading and thickness noise sources.

The Farassat 1A formulation for the acoustic pressure from thickness $\left(p_{T}\right)$ and loading $\left(p_{L}\right)$ noise sources at an observer location $\boldsymbol{x}$ and time $t$ is given by

$$
\begin{aligned}
& p_{T}(\boldsymbol{x}, t)=\frac{1}{4 \pi} \sum_{j}\left[\frac{\rho_{o} \dot{v}_{n}}{r\left(1-M_{r}\right)^{2}}\right]_{\mathrm{ret}, j} \Delta S_{j} \\
& +\frac{1}{4 \pi} \sum_{j}\left[\frac{\rho_{o} v_{n}\left(r \dot{M} \hat{r}_{i}+a_{o} M_{r}-a_{o} M^{2}\right)}{r^{2}\left(1-M_{r}\right)^{3}}\right]_{\mathrm{ret}, j} \Delta S_{j} \\
& p_{L}(\boldsymbol{x}, t)=\frac{1}{4 \pi a_{o}} \sum_{j}\left[\frac{l_{i} \hat{r}_{i}}{r\left(1-M_{r}\right)^{2}}\right]_{\mathrm{ret}, j} \Delta S_{j} \\
& +\frac{1}{4 \pi} \sum_{j}\left[\frac{l_{i} \hat{r}_{i}-l_{i} M_{i}}{r^{2}\left(1-M_{r}\right)^{2}}\right]_{\mathrm{ret}, j} \Delta S_{j} \\
& +\frac{1}{4 \pi a_{o}} \sum_{j}\left[\frac{l_{r}\left(r \dot{M}_{i} \hat{r}_{i}+a_{o} M_{r}-a_{o} M^{2}\right)}{r^{2}\left(1-M_{r}\right)^{3}}\right]_{\mathrm{ret}, j} \Delta S_{j}
\end{aligned}
$$

The blade is discretized into a number of flat panels of surface area $\Delta S_{j}$, and the contribution of each panel $j$ to the noise is recorded in retarded time (subscript "ret") and interpolated to the desired observer time. The retarded or source time is the time at which sound is emitted from the source. The observer time is the time when the sound emitted from a source reaches the observer location. It is also the time at which the acoutic pressure is calculated.

In Eqs. (1) and (2), $\rho_{o}, a_{o}$ represent the ambient density and speed of sound, respectively. $v_{n}$ is the velocity of the quarter-chord point of the panel, projected in a direction normal to the airfoil camber. $l_{i}$ is the sectional load in the $i$ direction; $M_{i}$ is the Mach number of the source in the $i$ direction; $r$ is the distance from the source to the observer; and $\hat{r}=r /|r| .(\cdot)$ denotes the rate of change of the quantity $(\cdot)$ with respect to source time.

As conceptualized by Schmitz [22], the forward section of an airfoil displaces fluid outward and acts as a pressure source, whereas the aft part of the airfoil acts as a pressure sink; this is illustrated in 


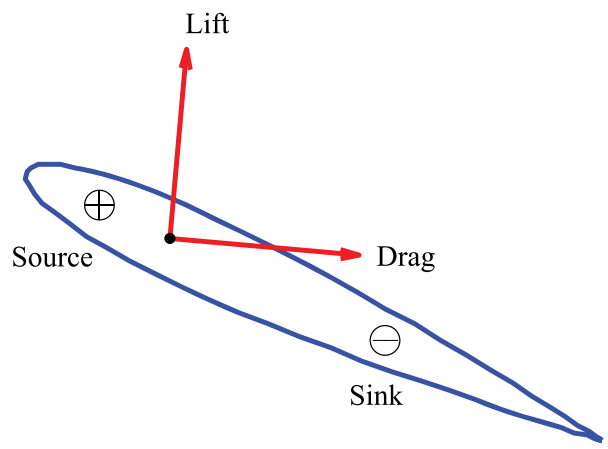

Fig. 1 Source/sink representation for thickness noise generation.

Fig. 1. In this paper, the mass displacement is represented by a single source at $x / c=1 / 8$ and a single sink at $x / c=5 / 8$ for each airfoil section. The sectional loads are assumed to be point forces collocated at $x / c=1 / 4$.

This compact acoustic model is a reasonable approximation for far-field noise calculations because the distance of the observer from the noise source is typically more than an order of magnitude larger than the blade chord. Extensive validation studies of thickness and loading noise signatures $[17,18,23,24]$ have been performed with this model. To further demonstrate and verify the accuracy of this formulation, the compact chord model is compared against a prediction based on the full geometry and blade pressure distribution data obtained from the SUmb/CDP CFD code [25]. The configuration chosen is the UH-60 rotor in high-speed forward flight [26] (advance ratio $\mu=0.3, M_{\text {tip }}=0.63$ ). Figure 2 shows a sample solution where the observer is located three rotor radii in front of the rotor plane, confirming the good agreement between the compact loading and full geometry models.

\section{Brief Review of the AVINOR Code}

Passive/active control simulations are performed using the Active Vibration and Noise Reduction (AVINOR) comprehensive rotorcraft analysis code, which consists of 1) a structural dynamic model that can represent a rotor blade undergoing moderate deflections with coupled flap-lag-torsional dynamics, 2) a nonlinear unsteady CFD based reduced-order model (ROM) [27] that captures the sectional aerodynamic loads accurately, and 3) a control model suitable for noise and vibration reduction. The code has been validated in previous studies $[13,28]$. The principal features of AVINOR are summarized next.

\section{A. Structural Dynamic Model}

The rotor is modeled as a four-bladed hingeless rotor, with fully coupled flap-lag-torsional dynamics for each blade. Two different structural models are used in this comparative study: a Galerkin-type finite element model for the rotor blade with swept tip for passive control, and a global Galerkin model for the straight blade with an OBC device for active control.

\section{Galerkin-Type Finite Element Model}

The finite element model is based on an analysis developed by Yuan and Friedmann [29,30], which is capable of modeling blades with transverse shear deformations, cross-sectional warping, and swept tips. The equations of motion are formulated using a finite element discretization of Hamilton's principle, with the assumption that the blade undergoes moderate deflections. The beam-type finite elements used for the discretization have 23 nodal degrees of freedom. In this study, modal reduction employing eight normal modes, namely the first three flap modes, first two lead-lag modes, first two torsional modes, and the first axial mode, are used to reduce the number of structural degrees of freedom.

\section{Global Galerkin Model}

For active control, the isotropic blade model originally developed by Millott and Friedmann [31] is used. The rotor blade is modeled as a slender, linear elastic, isotropic cantilever beam undergoing moderate deflections. The structural dynamic equations are discretized using the global Galerkin method, employing three flap, two lead-lag, and two torsional free vibration modes of the rotating blade. Each rotating mode is obtained from nine nonrotating uniform beam modes. The effect of control surfaces on the structural properties of the blade is neglected. Thus, the control surfaces influence blade behavior only through their effect on the aerodynamic and inertial loads. This structural model has a computational efficiency that is superior to the finite element model, particularly when coupled with OBC devices.

\section{B. Aerodynamic Model}

The blade/plain flap sectional aerodynamic loads for attached flow are calculated using a CFD-based reduced-order model (ROM) [32]. The ROM is based on rational function approximations (RFA) representing a least-squares fit to the aerodynamic load response data obtained using CFD simulations. This model accurately predicts the unsteady aerodynamic lift, moment, and drag forces while taking a fraction of computational time compared to CFD. Accounting for both unsteady lift and drag forces is critical for accurate in-plane noise prediction. For the swept tip region, the chordwise component of the freestream flow velocity at the swept blade section is used to determine the unsteady aerodynamic loads. The ROM model is linked to a free wake model [33] that yields a spanwise and azimuthally varying inflow distribution. For the separated flow regime, the aerodynamic loads are obtained using the ONERA dynamic stall model [34].
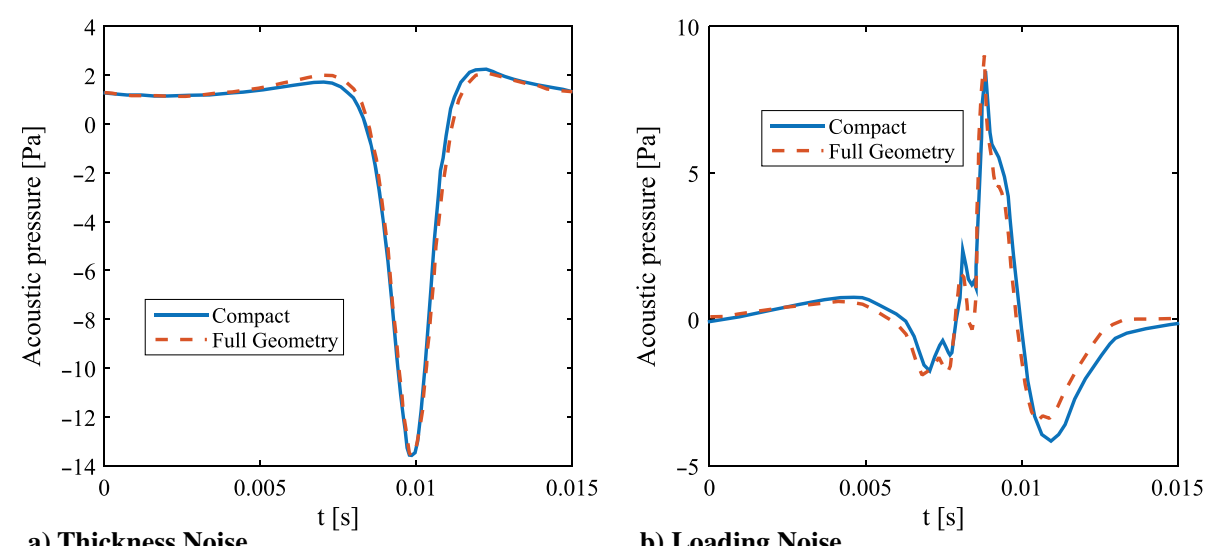

a) Thickness Noise

b) Loading Noise

Fig. 2 Verification of the compact model acoustic pressure predictions for a UH60 rotor at $\mu=0.30, M_{\text {tip }}=0.63$, at an observer located $3 R$ in front of the rotor plane. 


\section{Coupled Aeroelastic Response/Trim Solution}

For the passive control study, the equation of motion for the blade representing an equilibrium between the inertia, aerodynamic, and structural loads is discretized using a finite element approach. The finite element degrees of freedom are reduced by a normal mode transformation using coupled free vibration modes of the rotating blade. This process results in a system of coupled nonlinear differential equation with periodic coefficients that can be written as

$$
[M(\boldsymbol{y})] \ddot{\boldsymbol{y}}+[C(\boldsymbol{y}, \dot{\boldsymbol{y}})] \dot{\boldsymbol{y}}+[K(\boldsymbol{y}, \dot{\boldsymbol{y}}, \ddot{\boldsymbol{y}})] \boldsymbol{y}+\boldsymbol{F}(\boldsymbol{y}, \dot{\boldsymbol{y}}, \ddot{\boldsymbol{y}})=0
$$

This system of equations is then cast into the first-order state variable form and integrated in the time domain using the AdamsBashforth predictor-corrector algorithm. Two different trim procedures were used. For the validation studies, a wind-tunnel trim procedure, where only two moment equations (roll and pitch) are enforced, is used to replicate the test conditions. A propulsive trim procedure, where three force equations (longitudinal, lateral, and vertical) and three moment equations (roll, pitch, and yaw) are enforced, was employed in the verification studies and final results. The trim equations are solved in a coupled manner with the aeroelastic equations of motions [Eq. (3)]. A simplified tail rotor model, based on uniform inflow and blade element theory, is used.

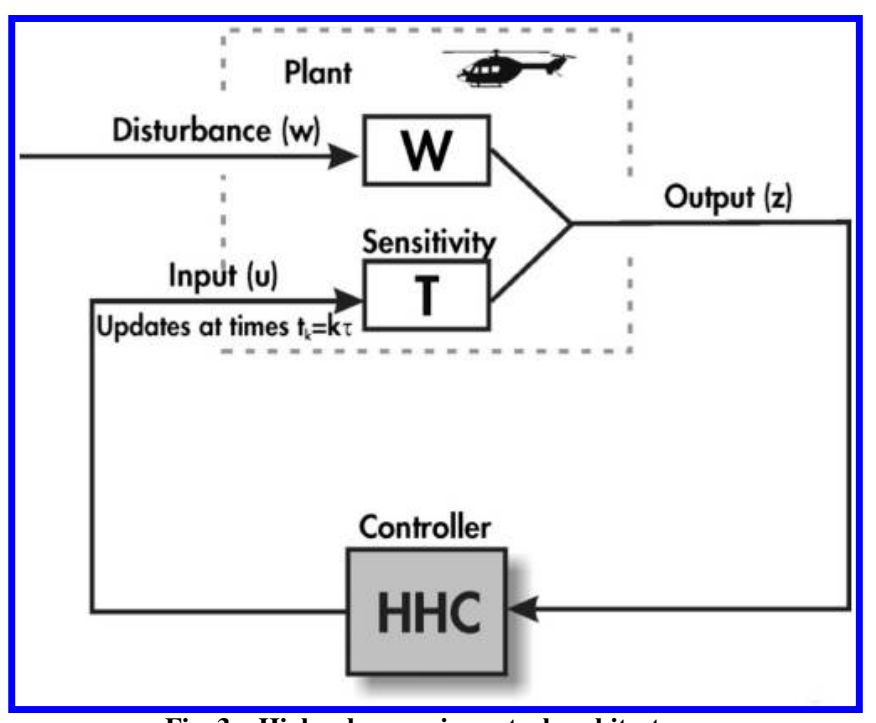

Fig. 3 Higher harmonic control architecture.
The six trim variables are the rotor shaft angle $\alpha_{R}$, the collective pitch $\theta_{0}$, the cyclic pitch $\theta_{1 s}$ and $\theta_{1 c}$, the tail rotor constant pitch $\theta_{0 t}$, and lateral roll angle $\phi_{R}$. The vibratory hub shears and moments are calculated by integrating the distributed inertial and aerodynamic loads over the entire blade span in the rotating frame, then transforming these loads to the hub fixed nonrotating system, and summing the contributions from each blade.

\section{Higher Harmonic Control Algorithm}

Active control of vibration and noise in this study is implemented using the adaptive HHC algorithm $[\underline{27}, \underline{35}]$. This algorithm is based on the assumption that the helicopter can be represented by a linear model relating the output of interest $\boldsymbol{z}$ to the control input $\boldsymbol{u}$. The measurement of the plant output and update of the control input are performed at specific times $t_{k}=k \tau$, where $\tau$ is the time interval between updates during which the plant output reaches a steady state. In actual implementation of the algorithm, this time interval may be one or more revolutions. A schematic of the HHC architecture implemented on a helicopter is shown in Fig. 3 .

The output vector at the $k$ th time step is given by

$$
z_{k}=\boldsymbol{T} \boldsymbol{u}_{k}+\boldsymbol{W} \boldsymbol{w}
$$

where the sensitivity matrix $\boldsymbol{T}$ represents a linear approximation of the helicopter response to the control and is given by

$$
\boldsymbol{T}=\frac{\partial z}{\partial u}
$$

The controller is based on the minimization of a general quadratic cost function:

$$
J\left(z_{k}, \boldsymbol{u}_{k}\right)=\boldsymbol{z}_{k}^{T} \boldsymbol{Q} z_{k}+\boldsymbol{u}_{k}^{T} \boldsymbol{R} \boldsymbol{u}_{k}
$$

The optimal control input is determined from the requirement

$$
\frac{\partial J\left(z_{k}, \boldsymbol{u}_{k}\right)}{\partial \boldsymbol{u}_{k}}=0
$$

which yields the optimal control law $\boldsymbol{u}_{k, \text { opt }}$, given by

$$
\boldsymbol{u}_{k, \mathrm{opt}}=-\left(\boldsymbol{T}^{T} \boldsymbol{Q T}+\boldsymbol{R}\right)^{-1}\left(\boldsymbol{T}^{T} \boldsymbol{Q}\right)\left(z_{0}-\boldsymbol{T} \boldsymbol{u}_{0}\right)
$$

This is the classical version of the HHC algorithm that yields an explicit relation for the optimal control input. An adaptive version of the HHC algorithm has been shown to perform better than the

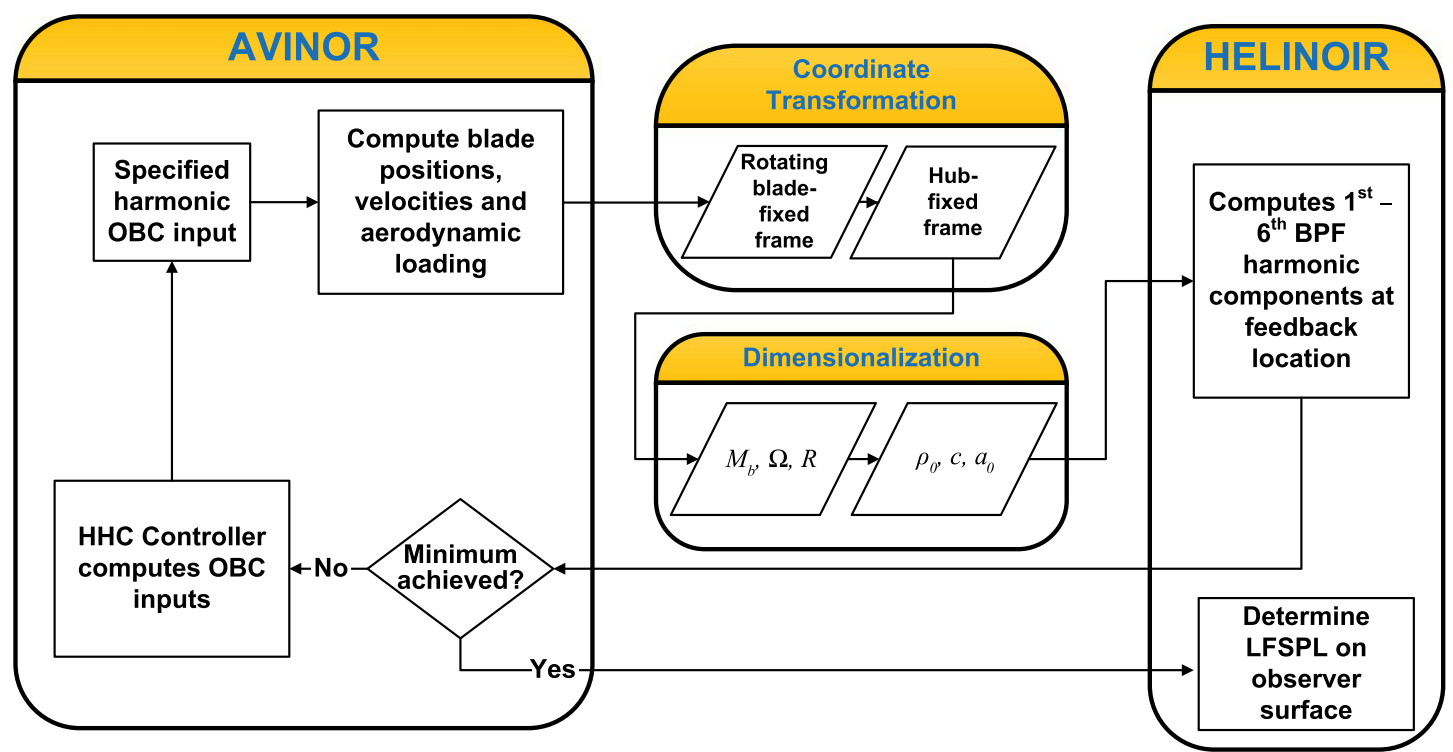

Fig. 4 Data flow between AVINOR and HELINOIR for active control. 
classical HHC when the model nonlinearities are significant, and the sensitivity matrix $\boldsymbol{T}$ is a poor approximation of the model [35]. In the adaptive HHC algorithm, the sensitivity matrix $\boldsymbol{T}$ is updated recursively, based on the input and output history, using a leastsquares methods. A detailed description of this version is provided in [35], and the details of its implementation for active in-plane noise control are provided in [16].

\section{Aeroelastic-Aeroacoustic Framework}

The data flow between the AVINOR and HELINOIR codes is illustrated by Fig. 4 for the case of active on-blade control. A set of coupled trim/aeroelastic equations is solved in AVINOR to determine the blade aeroelastic response and aerodynamic loading at each time step. The blade position and velocities as well as the aerodynamic loads are passed on to the HELINOIR code for acoustic computation.

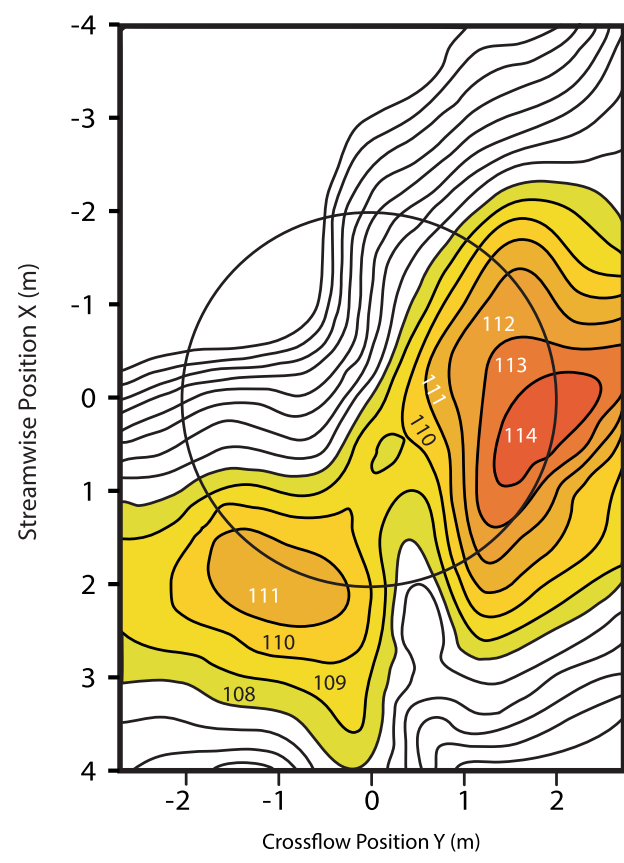

a) HART Experimental Results

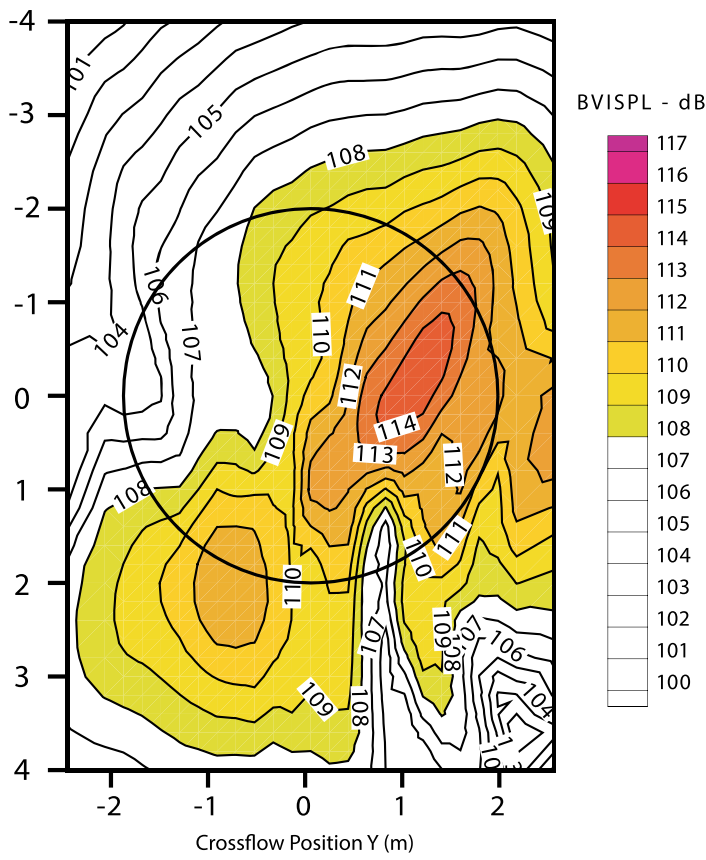

b) AVINOR/HELINOIR

Fig. 5 Validation of the acoustic computations against the HART experimental data.
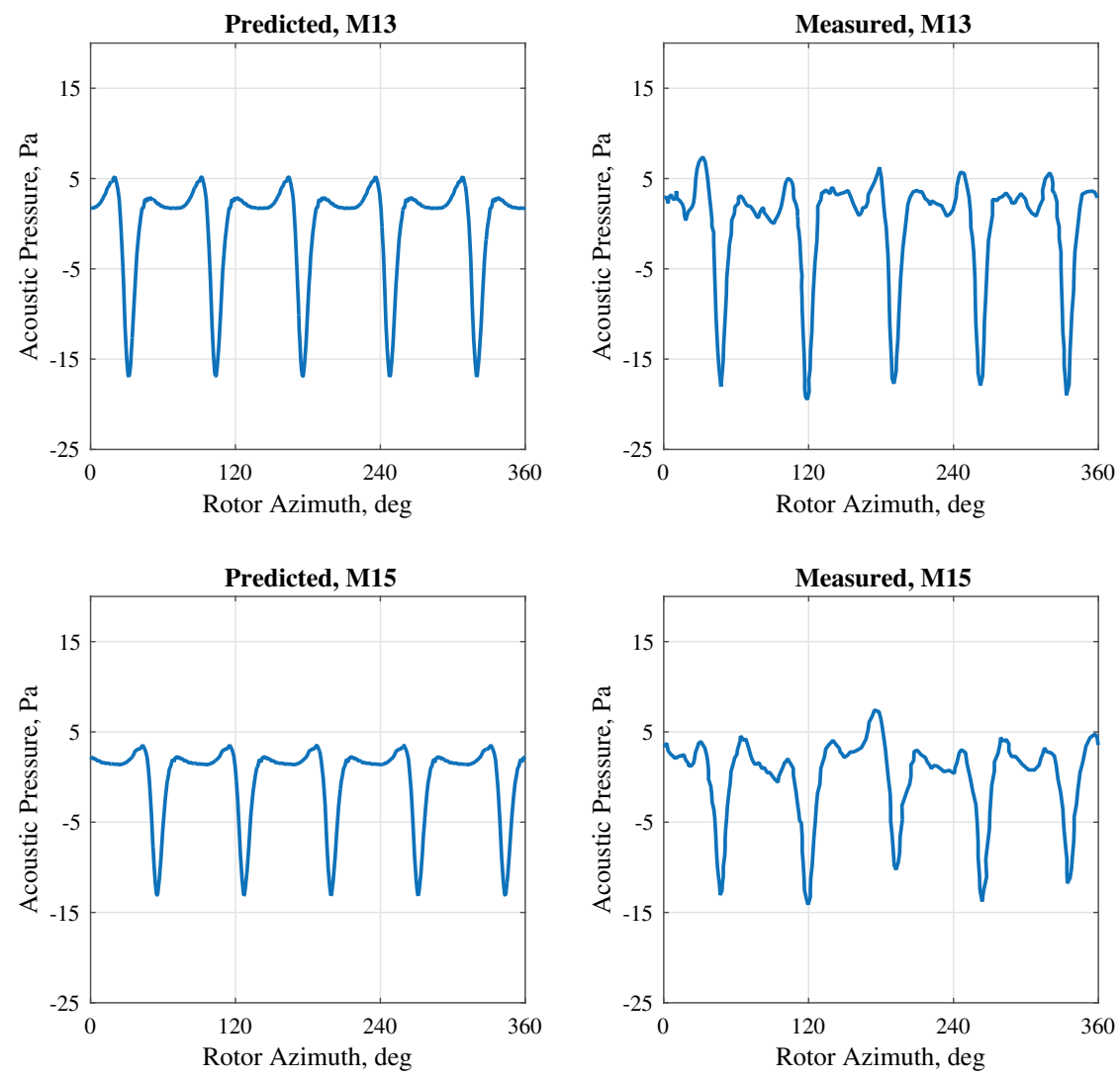

Fig. 6 Validation of the acoustic computations against the Boeing SMART experimental data (experimental data from [14]). 
To ensure data consistency between AVINOR and HELINOIR, a series of coordinate transformations and redimensionalizations is performed. The HELINOIR code computes the acoustic pressure, using the blade loads and kinematic data obtained from AVINOR, as a postprocessing step. For closed-loop active noise control, HELINOIR computes the acoustic pressure at the feedback location, which is sent back to the higher harmonic controller (HHC). The $\mathrm{HHC}$ algorithm computes the OBC inputs that minimize a quadratic cost function based on the feedback microphone noise levels as well as the control input magnitudes. For passive control, a similar framework is used, except that the feedback loop with the HHC is not present.

\section{Validation Studies}

The AVINOR/HELINOIR code, employing a straight blade modeled using the global Galerkin method, has been validated against experimental data [16] obtained in two major wind-tunnel tests: 1) the Higher-Harmonic-Control Aeroacoustic Rotor Test (HART) [36], and 2) the Boeing-SMART rotor wind-tunnel test conducted in the $40 \times 80 \mathrm{ft}$ wind tunnel at NASA Ames Research Center [37].

For the HART validation, the baseline flight condition corresponds to a heavy BVI descending flight, with $\mu=0.15, C_{T}=0.044$, and $\alpha_{D}=6.5 \mathrm{deg}$. The HART rotor is a $40 \%$ dynamically and Machscaled model of a four-bladed hingeless Messerschmitt-BölkowBlohm (MBB) BO-105 main rotor. The BVI sound pressure levels (BVISPLs) were measured on a carpet plane positioned $1.15 R$ below the rotor hub and parallel to the hub plane. The noise levels from the experiment are shown in Fig. $5 \mathrm{a}$, and the results from the simulation with AVINOR/HELINOIR are shown in Fig. 5b. The new code predicts the BVI noise levels on the carpet plane very well, capturing the magnitude and location of the noise peaks of $114 \mathrm{~dB}$ on the advancing side and $111 \mathrm{~dB}$ on the retreating side accurately.

For the Boeing-SMART rotor validation, the baseline flight condition was level cruise, at $\mu=0.30$ and $C_{T}=0.006$. The SMART rotor is a full-scale, bearingless, five-bladed main rotor modified from an MD900 Explorer rotor system. For the code validation, the structural properties of the blade model were chosen to match the fundamental frequencies $\omega_{L 1}, \omega_{F 1}, \omega_{T 1}$ of the SMART rotor [38], following the procedure described in [39]. Three in-plane microphones (M13, M15, and M14), positioned along a straight line originating from the advancing blade tip to the tunnel centerline, were used for low-frequency, in-plane noise measurements. The predicted and measured acoustic pressure histories at the M13 and M15 microphones are shown in Fig. 6 , and it is evident that the pressure history is predicted well, capturing the peak-to-peak amplitude accurately. Further details of the code validation and verification can be found in [16].

\section{Verification Studies}

To model the advanced tip geometry used in passive control, a beam-type finite element model of a rotor blade, based on the analysis in [30], was developed and implemented in AVINOR/HELINOIR as part of this study. To verify the new structural model, the acoustic and vibration calculations are compared against the results from the AVINOR/WOPWOP code [39] based on the global Galerkin method.

A helicopter configuration resembling a full-scale four-bladed MBB BO-105 hingeless rotor is used. The rotor parameters are listed in Table 1 and are also given in [40]. All the values in the table (except $C_{W}, \gamma$, and $\sigma$ ) have been nondimensionalized using $M_{b}, R$, and $1 / \Omega$ for mass, length, and time, respectively. Constant mass and stiffness distributions are assumed along the blade span. Using these parameters, it was found that six beam-type finite elements are needed to match the modal frequencies $\omega_{F i}, \omega_{L i}, \omega_{T i}$ used in the global Galerkin model. The baseline flight condition is a descending flight at an advance ratio $\mu=0.15$, thrust coefficient $C_{T}=0.005$, and descent angle $\alpha_{D}=6.5 \mathrm{deg}$, representing heavy BVI conditions. The acoustic environment represented by BVISPL
Table 1 Baseline rotor parameters

\begin{tabular}{lc}
\hline \hline Parameters & Values \\
\hline & Dimensional rotor data \\
$R$ & $4.91 \mathrm{~m}$ \\
$M_{b}$ & $27.35 \mathrm{~kg}$ \\
$\Omega$ & $425 \mathrm{rpm}$ \\
\multicolumn{1}{c}{ Nondimensional rotor data } \\
$N_{b}$ & 4 \\
$L_{b}$ & 1.0 \\
$c / R$ & 0.05498 \\
$\theta_{t w}$ & $-8 \mathrm{deg}$ \\
$e$ & 0 \\
$X_{A}$ & 0 \\
$X_{I b}$ & 0 \\
$\omega_{F}$ & $1.124,3.40,7.60$ \\
$\omega_{L}$ & $0.732,4.458$ \\
$\omega_{T}$ & $3.17,9.08$ \\
$\gamma$ & 5.5 \\
$\sigma$ & 0.07 \\
$\beta_{p}$ & $2.5 \mathrm{deg}$ \\
$C_{W}$ & \\
$C_{d f}$ & 0.005 \\
$X_{F A}$ & 0.031 \\
$Z_{F A}$ & 0.0 \\
$X_{F C}$ & 0.3 \\
$Z_{F C}$ & 0.0 \\
$\mu$ & 0.3 \\
$M_{\infty}$ & 0.3 \\
$M_{\text {tip }}$ & 0.191 \\
$\alpha_{R}$ & 0.637 \\
$\theta_{0}$ & \\
$\theta_{1 c}$ & $-5.50 \mathrm{deg}$ \\
$\theta_{1 s}$ & $8.76 \mathrm{deg}$ \\
$\theta_{0 t}$ & $0.606 \mathrm{deg}$ \\
$\phi_{R}$ & $-5.08 \mathrm{deg}$ \\
\hline \hline & $2.30 \mathrm{deg}$ \\
& $3.08 \mathrm{deg}$ \\
\hline
\end{tabular}

computed on a carpet plane located $1.15 R$ beneath the rotor is compared in these verification studies.

The baseline noise level on the carpet plane computed using the AVINOR/WOPWOP code employing the global Galerkin model is shown in Fig. 7a. Results from simulations performed using the finite element method (FEM) AVINOR code in combination with WOPWOP and HELINOIR are shown in Figs. $7 \mathrm{~b}$ and $7 \mathrm{c}$, respectively. It is evident that the finite element model, whether it is coupled with WOPWOP or HELINOIR, predicts the BVI noise levels on the carpet plane well, capturing the magnitude and location of the noise peaks of $114 \mathrm{~dB}$ on the advancing side and $111 \mathrm{~dB}$ on the retreating side.

The associated baseline 4/rev nondimensional vibratory hub loads for the three codes are shown in Fig. $\underline{8}$. There is good agreement in all six components of the vibratory hub loads. It is evident that both the acoustic and vibratory load predictions from the finite element structural model, coupled with HELINOIR, agree well with the global Galerkin AVINOR/WOPWOP code combination. The differences in vibrations obtained from the FEM/AVINOR/ WOPWOP and FEM/AVINOR/HELINOIR are attributed to the differences in the aerodynamic models used. The FEM/AVINOR/ WOPWOP employs a double-lattice-based RFA aerodynamic model [15], whereas the FEM/AVINOR/HELINOIR employs a CFD-based RFA model [32].

\section{Results and Discussion}

The simulation results presented in this section are for a helicopter resembling a full-scale four-bladed MBB BO-105 hingeless rotor in level flight at an advance ratio $\mu=0.3$. The baseline rotor parameters are provided in Table 1. The rotor is trimmed using propulsive trim.

Passive in-plane noise control is implemented through a sweep, dihedral, or anhedral tip of the segment of the blade representing the outboard $10 \%$ of the blade. The orientation of the tip sweep relative to 


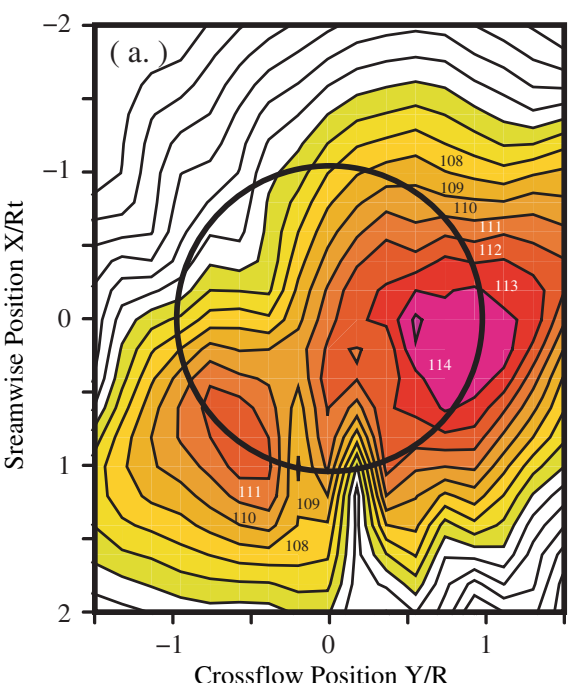

a) Global Galerkin/AVINOR/WOPWOP

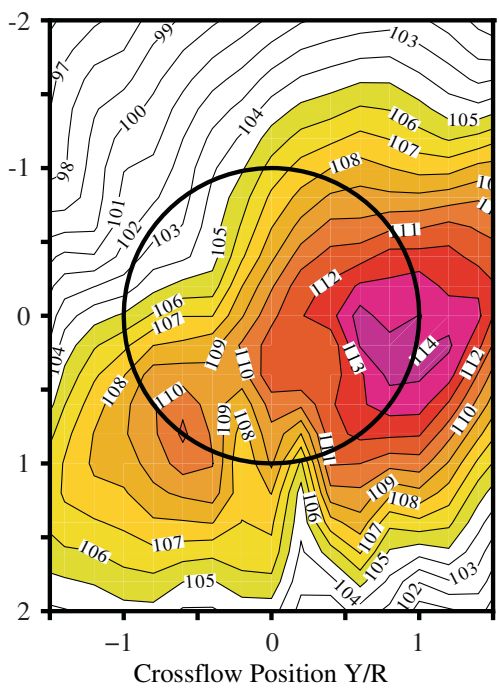

b) FEM/AVINOR/WOPWOP

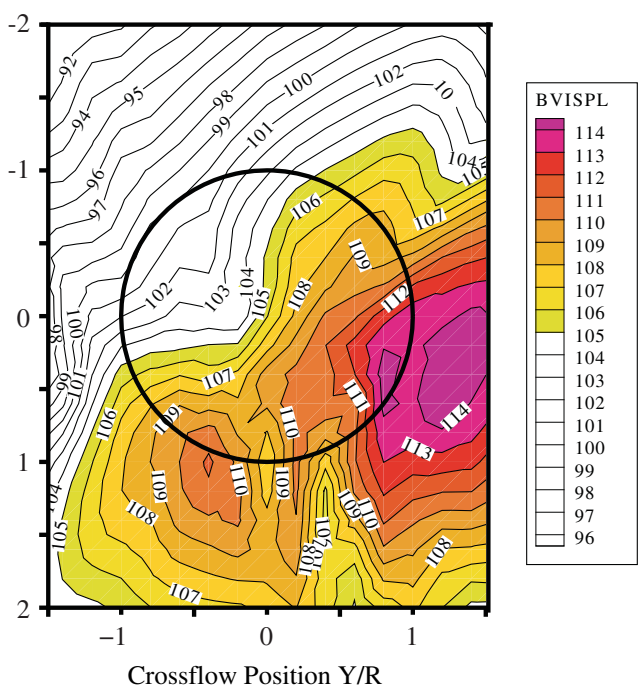

c) FEM/AVINOR/HELINOIR

Fig. 7 Comparison of the noise levels from a straight blade computed on the carpet plane employing a) a global Galerkin model, and b-c) Galerkin-type FEM, at $\mu=0.15, C_{T}=0.005$, and $\alpha_{D}=6.5 \mathrm{deg}$.

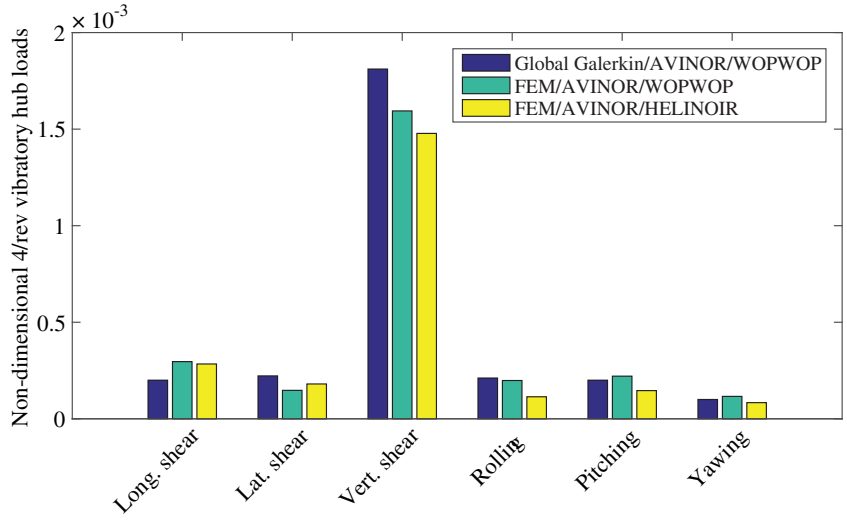

Fig. 8 Comparison of the vibration levels from a baseline blade employing a global Galerkin model and Galerkin-type FEM at $\boldsymbol{\mu}=\mathbf{0 . 1 5}$, $C_{T}=0.005$, and $\alpha_{D}=6.5 \mathrm{deg}$.

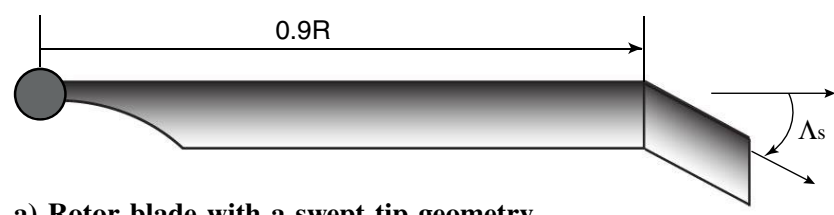

a) Rotor blade with a swept tip geometry.

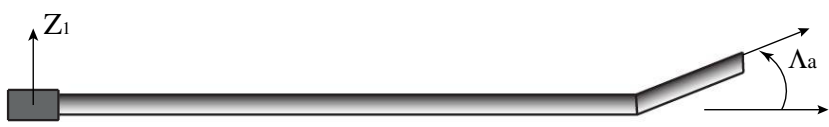

b) Rotor blade with a anhedral/dihedral tip geometry.

Fig. 9 Rotor blade with passive control.

the straight portion is described by a sweep angle $\Lambda_{s}$ and is defined positive backward, as shown in Fig. 9a. The tip inclination angle from the horizontal $\Lambda_{a}$ is defined positive upward, as shown in Fig. 9b; therefore, $\Lambda_{a}>0$ deg for a dihedral tip and $\Lambda_{a}<0$ deg for an anhedral tip. The blade is modeled by six beam-type finite elements along the elastic axis. Five elements are used to model the straight segment of the blade, which spans $90 \%$ of the blade radius, and the tip is modeled using a single finite element. A range of sweep, dihedral, and anhedral angles up to a maximum of 6 deg was considered. The three tip geometry modifications considered in this study were

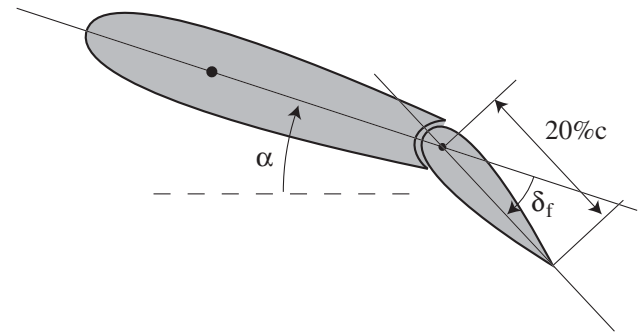

Fig. 10 On-blade control device used in the study.

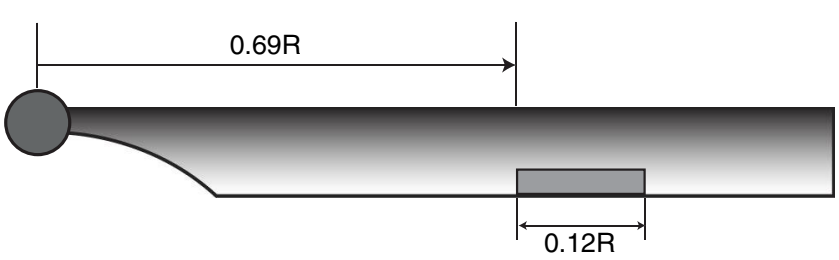

Fig. 11 Single-flap configuration of the plain flap on the rotor blade.

implemented, independently, one at a time. Dealing simultaneously with combined tip modifications such as sweep combined with anhedral was beyond the scope of this study because it would have required the use of an optimization framework.

Passive control results are compared against active control results obtained using a $20 \% \mathrm{c}$ plain flap, shown in Fig. 10. A single-flap configuration is used, shown in Fig. 11. The single flap has a span of $0.12 R$ and is centered at $0.75 R$. The rotor blade in the active control case is modeled as a slender beam cantilevered at the hub.

It was found in [16] that a microphone located on the left boom position provided the best feedback for closed-loop in-plane noise reduction. The feedback microphone location is illustrated in Fig. 12.

The far-field acoustic environment in front of the helicopter is characterized by low-frequency sound pressure level (LFSPL), consisting of the first through sixth blade-passage frequency (BPF) harmonic components of the rotor noise, which are the principal components of in-plane low-frequency noise [14]. The LFSPL is computed on a spherical segment located at a distance of $10 R$ in front of the rotor hub, with an azimuth angle between 135 and $225 \mathrm{deg}$ and an elevation angle between -90 and $15 \mathrm{deg}$, as shown in Fig. 13. This surface includes the observer locations in the forward in-plane direction where noise reduction is considered. For selected observer 


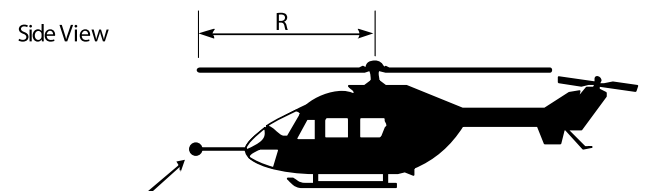

Onboard Microphone

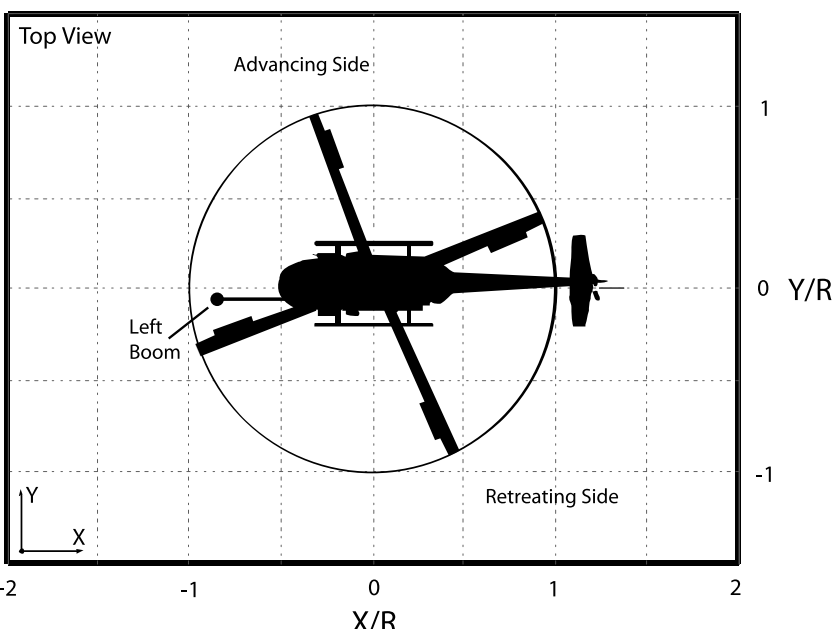

Fig. 12 Near-field onboard microphone feedback location on left boom and tip of the right skid.

locations of interest, the midfrequency sound pressure level (MFSPL) is used to characterize the higher-frequency components of the in-plane far-field noise. The MFSPL consists of the harmonic components greater than the sixth BPF.

\section{A. Noise Reduction Using Passive Control}

The baseline LFSPL noise obtained using the finite-element based AVINOR/HELINOIR code combination is shown in Fig. 14a. Two regions of high noise levels, above $95 \mathrm{~dB}$, are predicted in front of the rotor, at around $((y / r),(z / r))=(2,0)$ and $((y / r),(z / r))=$ $(7,-6)$. It is this directivity and the low-frequency content of the LFSPL noise that increases the range at which the helicopter can be detected in forward flight.

The LFSPL obtained using a rotor blade with swept tip $\left(\Lambda_{s}=6 \mathrm{deg}\right)$ is shown in Fig. 14b. The change in the LFSPL from the baseline with the introduction of swept tip is shown in Fig. 15a. Similarly, the change in the LFSPL with a dihedral tip $\left(\Lambda_{a}=6 \overline{\mathrm{deg}}\right)$ is shown in Fig. 15b, and the change in the LFSPL with an anhedral tip $\left(\Lambda_{a}=-6\right.$ deg $)$ is shown in Fig. 15c.

For the case of the swept tip, Fig. 15 a, there is no significant change in the LFSPL in the in-plane direction on the horizon, $(z / R)=0$ or 0 deg elevation angle. Therefore, the swept tip does not contribute significantly to the in-plane noise on the horizon at moderate tip speed when quadrupole noise is not significant. This result is in agreement with [6]. However, the swept tip reduces noise by up to $2 \mathrm{~dB}$ above the horizon, $(z / R)=2$ or $15 \mathrm{deg}$ elevation angle and more significantly causes a noise increase of up to $4 \mathrm{~dB}$ below the horizon, $(z / R)=-7$ or -45 deg elevation angle. Similarly, for the anhedral tip, Fig. $15 \mathrm{c}$, there is a noise reduction of up to $3 \mathrm{~dB}$ above the horizon and a noise increase of up to $6 \mathrm{~dB}$ below the horizon. This increase in the LFSPL noise below the horizon increases the range at which rotorcraft can be detected and is not desirable. On the other hand, a dihedral tip (Fig. 15b) achieved a noise reduction of up to $2 \mathrm{~dB}$ for $(z / R)>-6$, or an elevation angle above $-37 \mathrm{deg}$. This direction corresponds to the forward, slight downward tilt of the main rotor tip path plane and is significant for reducing the range at which the helicopter can be detected. However, a noise increase of up to $3 \mathrm{~dB}$ is also generated in the left out-of-plane direction. These results suggest

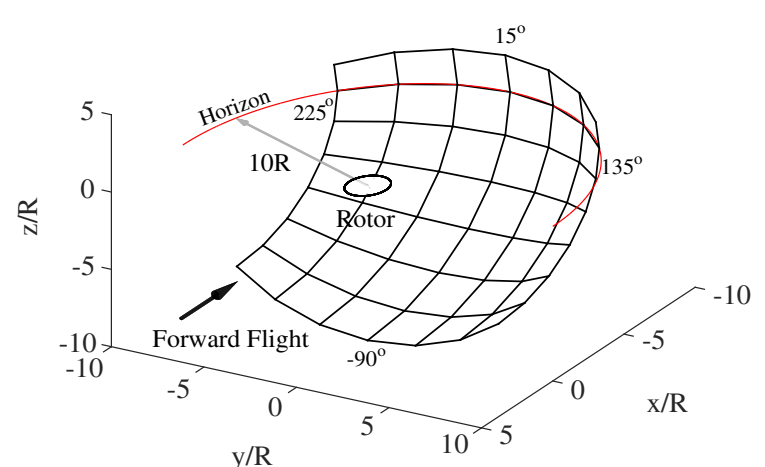

a) 3D View

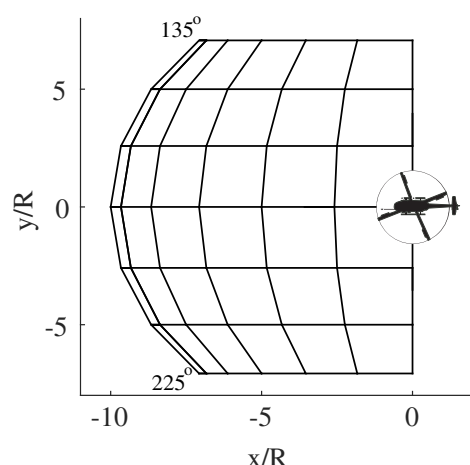

b) Top View

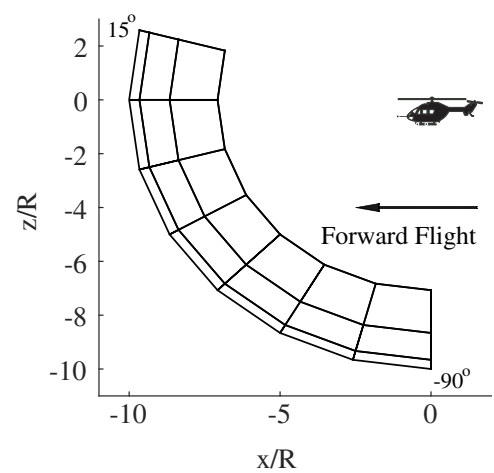

c) Side View

In-plane observer surface located at $10 R$ in front of the helicopter. Azimuthal variation is shown in Fig. 13b, and elevational variation is shown in Fig. 13c.

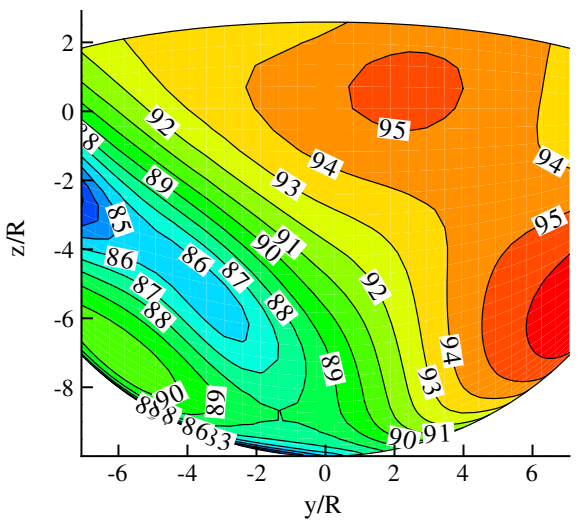

a) Baseline

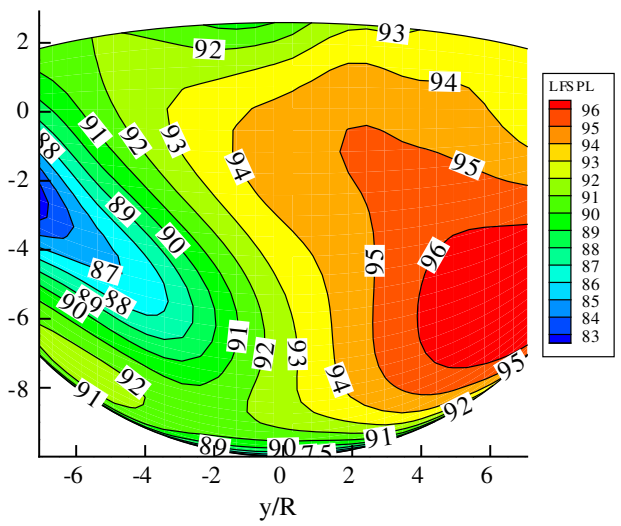

b) Passive control, $\Lambda s=6^{\circ}$

Fig. 14 In-plane LFSPL noise levels obtained using a baseline blade and blade with tip sweep, $\Lambda=6$ deg, on the observer surface at $10 R, \mu=0.3$. 


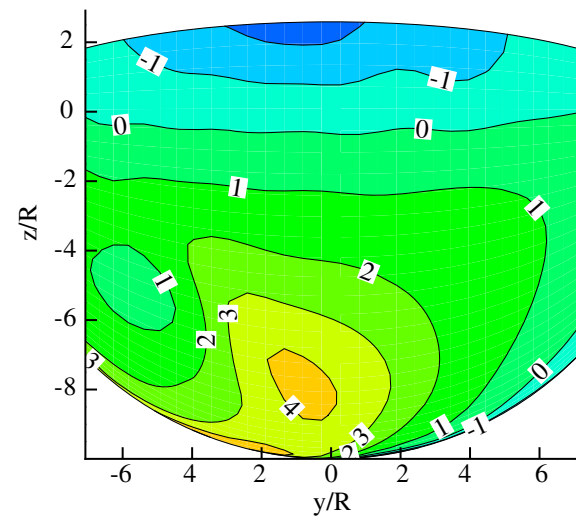

a) Sweep, $\Lambda s=6^{\circ}$

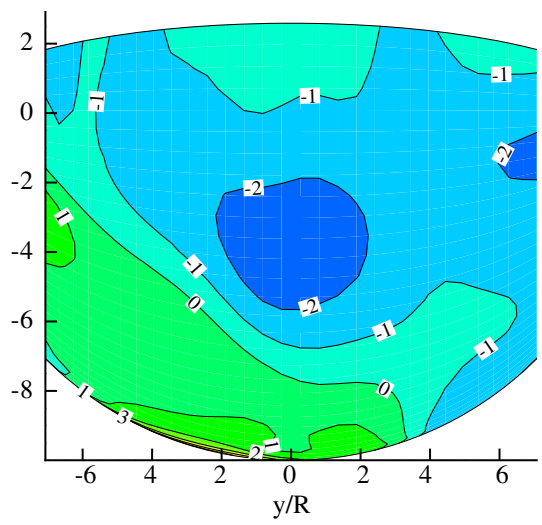

b) Dihedral, $\Lambda \mathrm{a}=6^{\circ}$

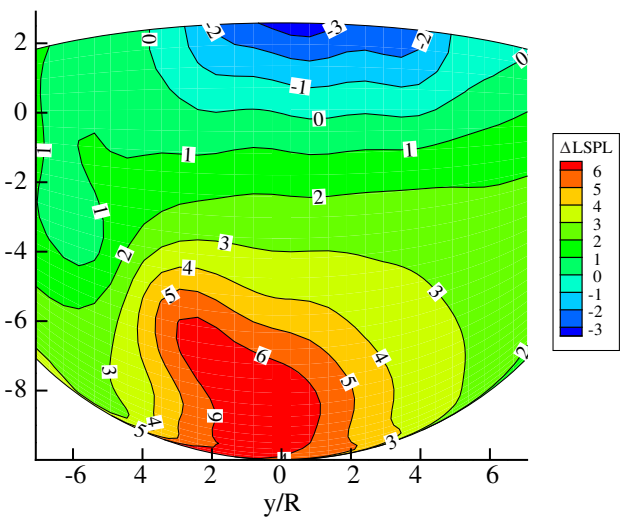

c) Anhedral, $\Lambda \mathrm{a}=-6^{\circ}$

Fig. 15 Change in the LFSPL obtained at $10 R$ for different tip geometries at $\mu=0.3$.

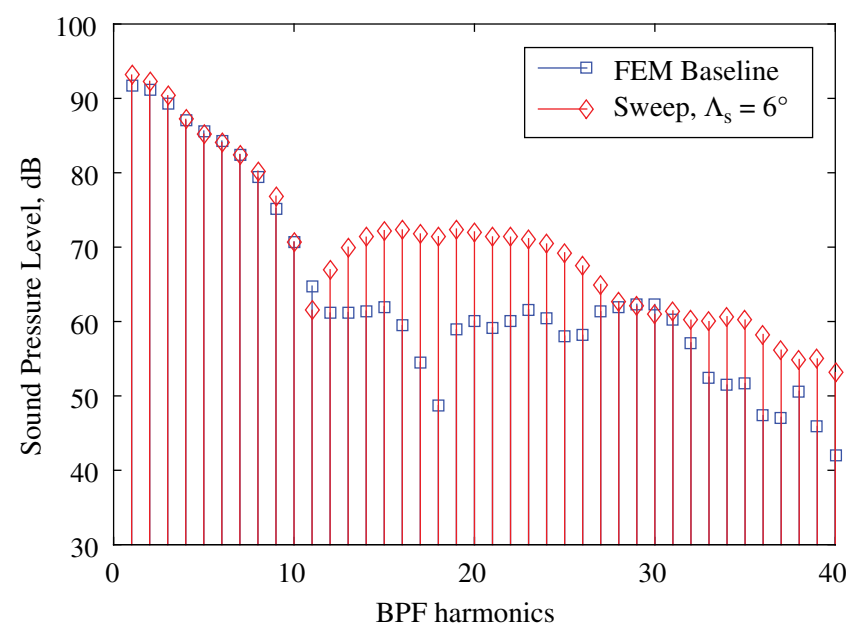

Fig. 16 Sound pressure level before and after passive control implemented through a swept tip.

that the reduction of in-plane LFSPL noise can result in a severe noise penalty in the out-of-plane direction.

The sound pressure levels of the first through 40th BPF harmonics for the baseline blade and the controlled case using a swept tip are shown in Fig. 16. The SPL is computed at the observer location, $((x / R),(y / R), \overline{(z} / R))=(-9.70,-0.27,-2.41)$, which corresponds to the forward, slight downward tilt of the main rotor tip path plane. This observer location is significant for determining the range at which the helicopter can be detected and is the point where maximum LFSPL reduction is achieved using active control [16]. The insertion loss is defined as the reduction in the sound pressure level due to the insertion of an active/passive control device. This is obtained by taking the difference between the SPL of the baseline and controlled case in Fig. 16. The insertion loss at the specified observer location due to the sweep, dihedral, and anhedral tip is shown in Fig. 17. It is evident that the reduction in LFSPL for the dihedral tip (Fig $\overline{15}$ b) is reflected in the insertion loss of the first through sixth BPF harmonics in Fig. 17b. Conversely, the increase in LFSPL for the swept and anhedral tip (Figs. 15a and 15c) is reflected in the negative insertion loss, or insertion gain, of the first through sixth BPF harmonics in Figs. $17 \mathrm{a}$ and $17 \mathrm{c}$. In all three cases, there is a overall insertion gain in the MFSPL, graeter than sixthBPF harmonic components, which is the frequency range of BVI noise. This suggests that passive control can add acoustic energy to the higher BPF harmonic components, contributing to near-field noise.

The reason behind in-plane noise increase/decrease below the horizon can be better understood by comparing the acoustic pressure history at an in-plane observer location for a rotor blade with and without tip geometry modification. Figure $18 \mathrm{a}$ shows the acoustic pressure history for the baseline blade at the observer location, $((x / R),(y / R),(z / R))=(-9.70,-0.27,-2.41)$. Figures $18 \mathrm{~b}-18 \mathrm{~d}$ show the acoustic pressure history with tip geometry modifications at the same location. In all four cases, there are only marginal changes in the thickness noise. For the tip sweep and tip anhedral, there is an increase in the loading noise component in phase with the negative peak thickness noise component, resulting in an overall increase in the magnitude of the total acoustic pressure and in-plane LFSPL noise below the horizon. On the other hand, for a tip dihedral, there is an out-of-phase increase in the loading noise component, which cancels the negative peak thickness noise, resulting in an overall decrease in the magnitude of the total acoustic pressure.

Figure 19 shows the associated $4 /$ rev vibratory hub loads for the three tip geometries compared to the baseline blade. There is a reduction in all six components of the vibratory hub loads for the swept and dihedral tip. For a tip sweep $\left(\Lambda_{s}=6 \mathrm{deg}\right)$, the vertical hub shear was reduced by up to $32 \%$, and for tip dihedral $\left(\Lambda_{a}=6 \mathrm{deg}\right)$,

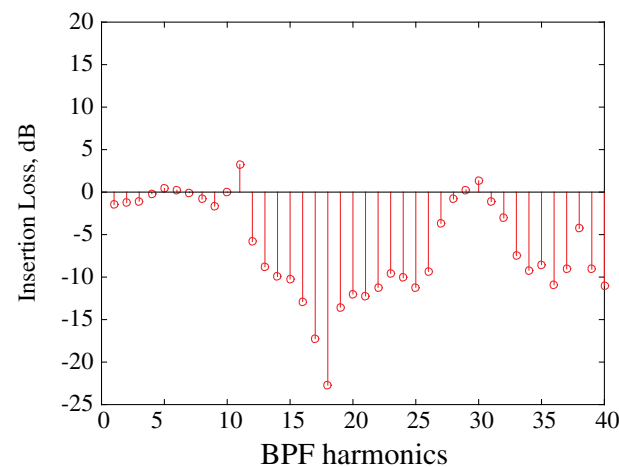

a) Sweep, $\Lambda s=6^{\circ}$

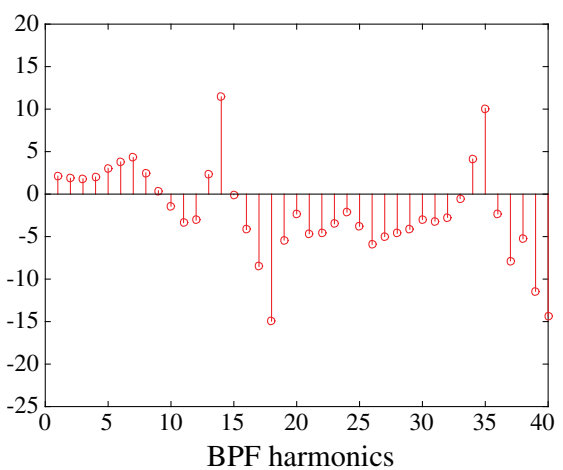

b) Dihedral, $\Lambda \mathrm{a}=6^{\circ}$

Fig. 17 Insertion loss due to passive control.

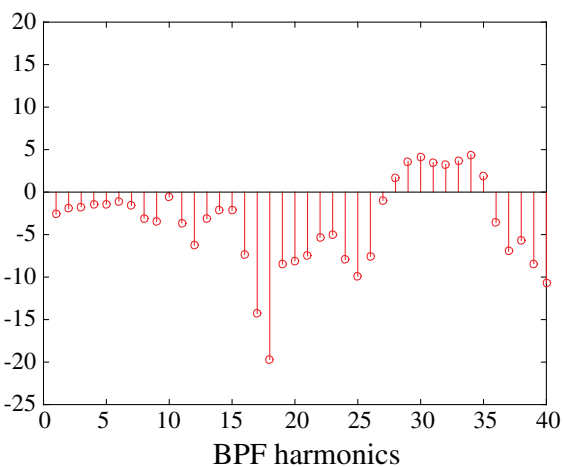

c) Anhedral, $\Lambda \mathrm{a}=-6^{\circ}$ 


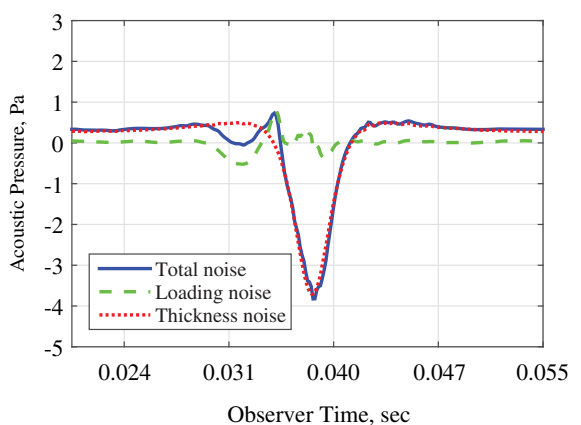

a) Baseline

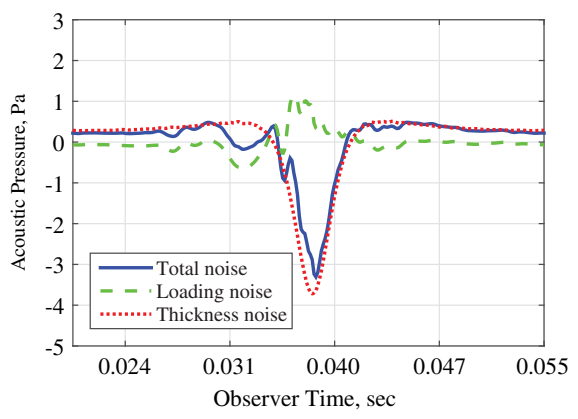

c) Dihedral, $\Lambda_{\mathrm{a}}=6^{\circ}$

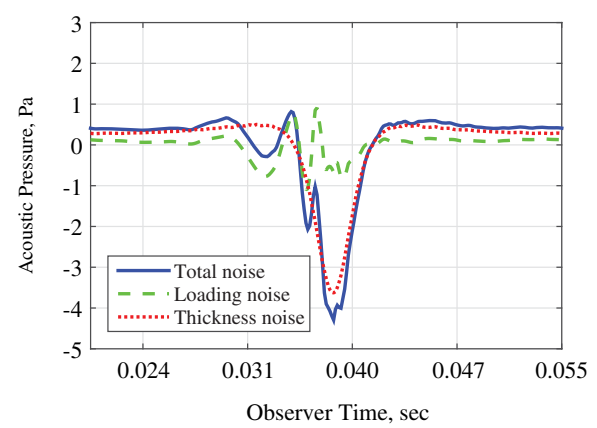

b) Sweep, $\Lambda_{\mathrm{s}}=6^{\circ}$

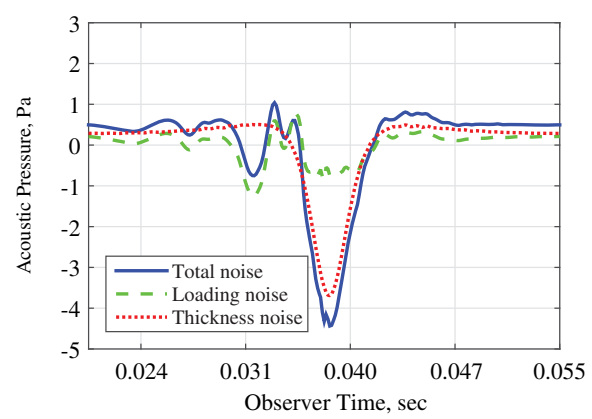

d) Anhedral, $a=-6^{\circ} \Lambda$

Fig. 18 Acoustic pressure histories at observer location $((x / R),(y / R),(z / R))=(-9.70,-0.27,-2.41)$ with a) baseline blade, b) swept tip, c) dihedral tip, and d) anhedral tip.

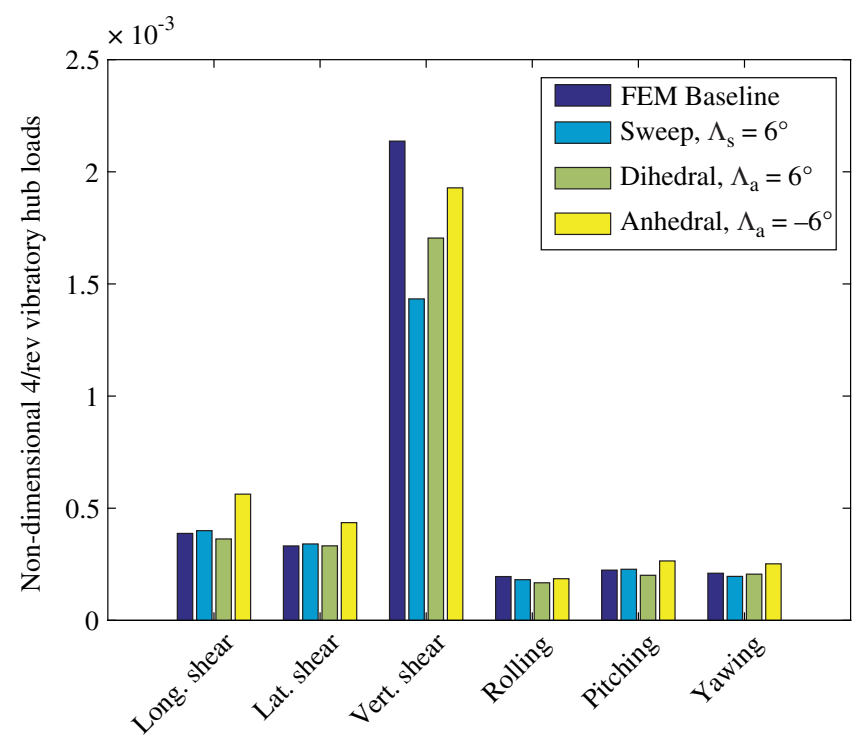

Fig. 19 Vibration levels for different tip geometries at $\boldsymbol{\mu}=\mathbf{0 . 3}$. the vertical hub shear was reduced by up to $19 \%$. For tip anhedral, there is a reduction in the vertical hub shear of up to $9 \%$; however, this was accompanied by an increase in the other vibratory hub load components. The reduction in the vibratory vertical hub shear, with the introduction of a tip sweep or tip anhedral, is consistent with the findings in [41].

From the simulation results performed, it is evident that a judicious choice of tip sweep and dihedral is required to obtain the optimum benefits of in-plane LFSPL noise and vibration reduction. A tip dihedral reduces in-plane noise with a reduction in vibratory hub loads. A tip sweep results in a maximum reduction in vibratory hub loads. Although not simulated in this study, a tip sweep is also beneficial in delaying and therefore reducing HSI noise at high advance ratio. A tip anhedral resulted in an increase in the in-plane LFSPL noise accompanied by an increase in the longitudinal, latitudinal shears and moments, and a relatively marginal reduction in the vertical hub shears compared to the other passive control means. It is interesting to note that, in the blade shape optimization study performed in [42], the blade design that improves aerodynamic performance and reduces overflight noise during forward flight is also one with backward sweep and mild dihedral.

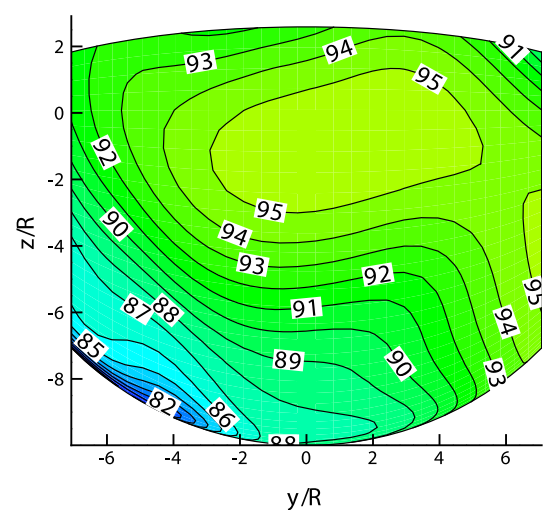

a) Baseline

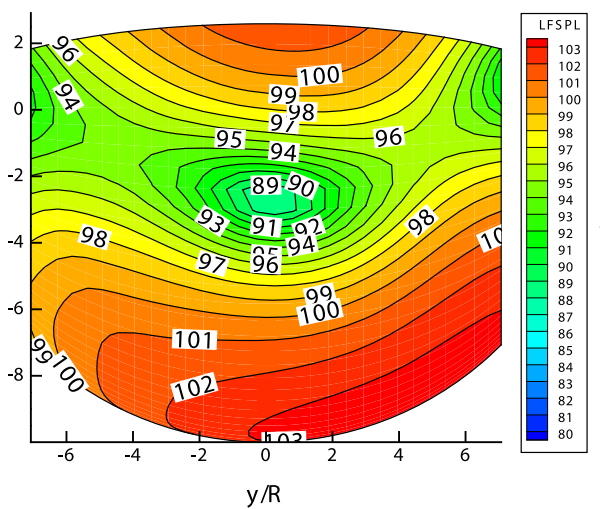

b) LFSPL during active control

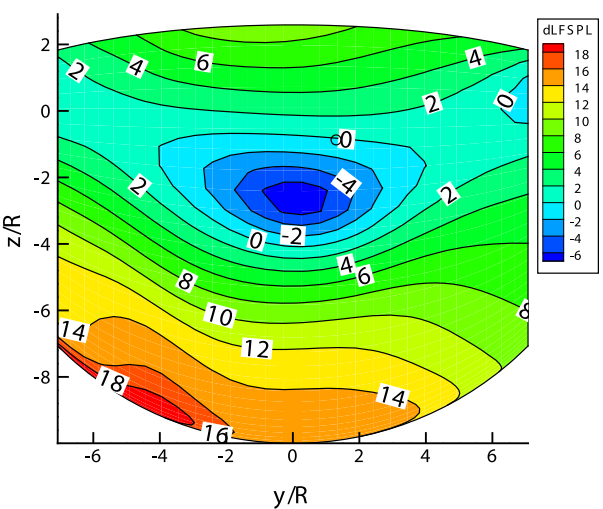

c) Change in LFSPL

Fig. 20 Baseline and the reduced in-plane noise levels obtained using active control, implemented using a single plain flap and a left nose boom feedback, for an MBB BO105 rotor during level flight, $\mu=0.30$. 


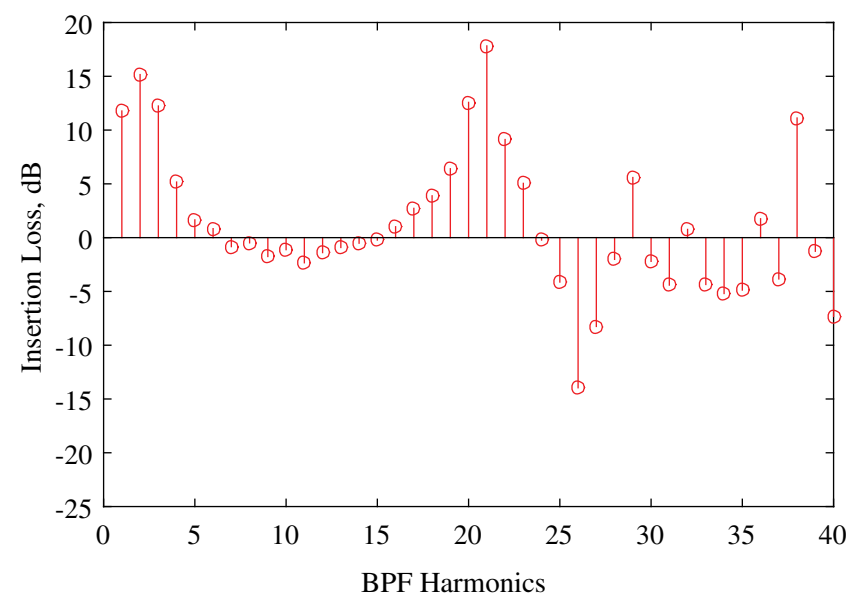

Fig. 21 Insertion loss due to active control.

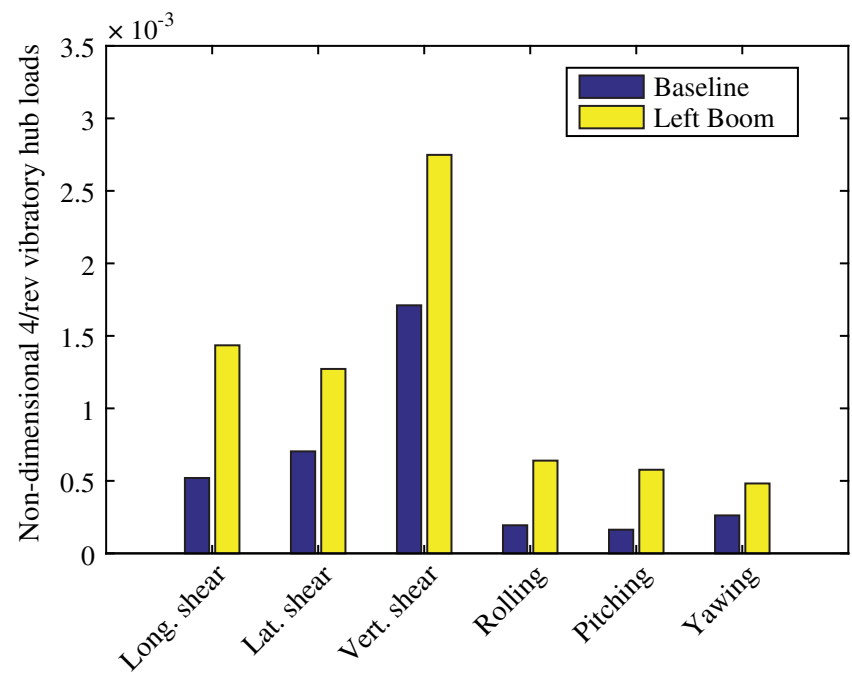

Fig. 22 Vibration penalty during in-plane noise reduction using a single plain flap with a left nose boom feedback.

\section{B. Noise Reduction Using Active Control}

The baseline LFSPL noise, without active control is shown in Fig. 20a. A region of high noise levels, above $95 \mathrm{~dB}$, is predicted around $((y / r),(z / r))=(0,0)$, that is, at 0 deg elevation angle and $180 \mathrm{deg}$ azimuth angle. This baseline is similar to that predicted using the finite element model shown in Fig. 14a. The differences could be attributed to the differences in the implementation of the wake model. The wake model used with the active control model was further modified to account for a higher wake resolution and the dual-vortex structure $[39,43]$.

The LF SPL noise reduction obtained using a single plain flap with a single near-field feedback microphone on the left boom is shown Fig. 20b. The change in LFSPL from the baseline is shown in Fig. $20 \mathrm{c}$. It can be seen that significant noise reduction of up to $6 \mathrm{~dB}$ was achieved between $-4 \leq(y / R) \leq 4,-4 \leq(z / R) \leq-1$ or an elevation angle between -23 and $4 \mathrm{deg}$ from the horizon and azimuth angle between 156 and $204 \mathrm{deg}$. This direction corresponds to the forward, slight downward tilt of the main rotor tip path plane and is the most significant for reducing the range at which the helicopter can be detected. However, a noise increase of up to $18 \mathrm{~dB}$ is generated in the left, out-of-plane location. This also suggests that the reduction of in-plane noise using active control can produce a severe noise penalty in the out-of-plane direction.

The insertion loss at the observer location, $((x / R),(y / R),(z / R))=$ $(-9.70,-0.27,-2.41)$, with active control is shown in Fig. 21 . It is evident that the insertion loss in the first through sixth BPF harmonics contributed to the in-plane LFSPL reduction. Unlike the insertion gain at the greater than sixth BPF harmonics due to passive control (Fig. 17), there is also significant insertion loss at the higher harmonics from the 16th to 23rd BPF harmonics. This means that active control is capable of noise reduction over a broader audible frequency range.

The vibration levels obtained during active in-plane noise control using the single plain flap are compared to the baseline levels in Fig. 22. An increase is noted in all six $4 /$ rev vibratory hub load components, with a maximum increase of $60.6 \%$ in the vertical hub shear.

\section{Comparison Between Passive and Active Control}

By comparing the change in LFSPL due to passive control (Fig. 15) and active control using a single plain flap (Fig. 20c), it can be seen that the reduction of in-plane LFSPL below the horizon can best be achieved using active control where a reduction of up to $6 \mathrm{~dB}$ was achieved. On the other hand, an LFSPL reduction of only $2 \mathrm{~dB}$

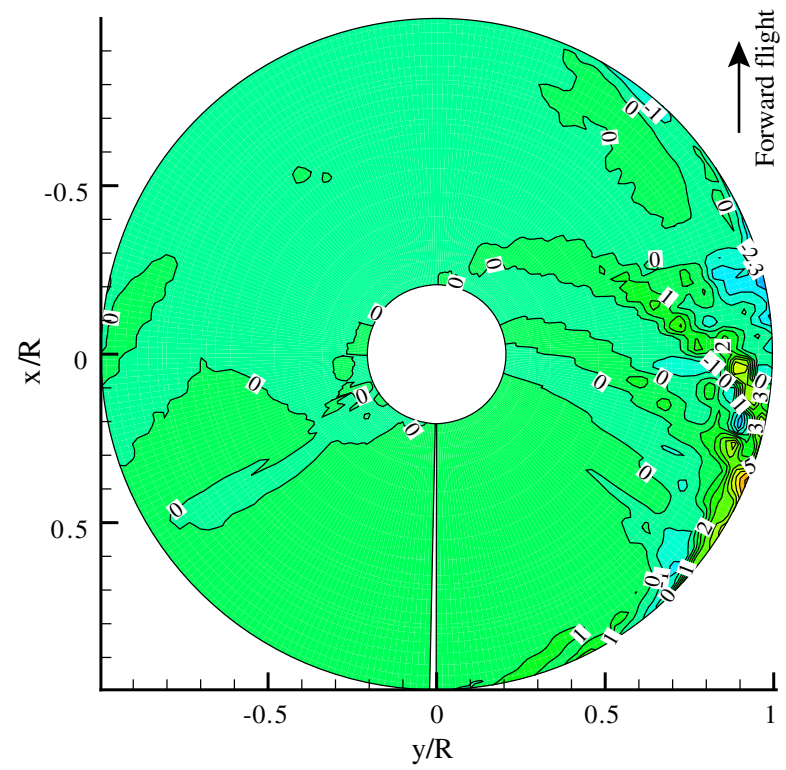

a) Baseline
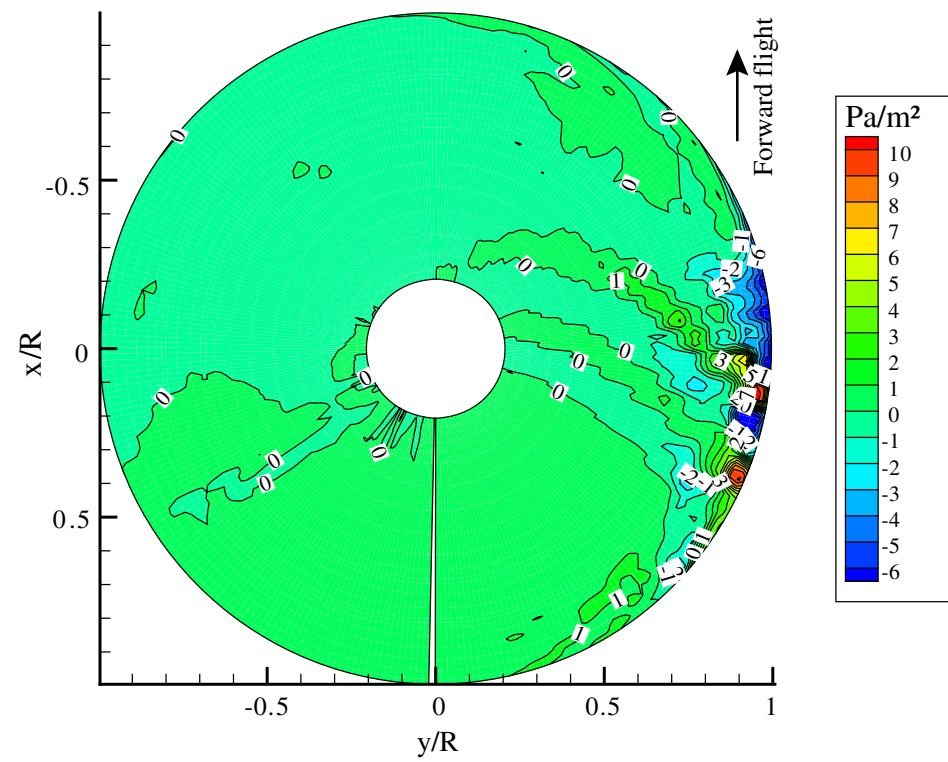

b) Sweep, $\Lambda s=6^{\circ}$

Fig. 23 Loading noise source density of baseline blade and blade with tip sweep, $\Lambda_{s}=6 \mathrm{deg}$. 


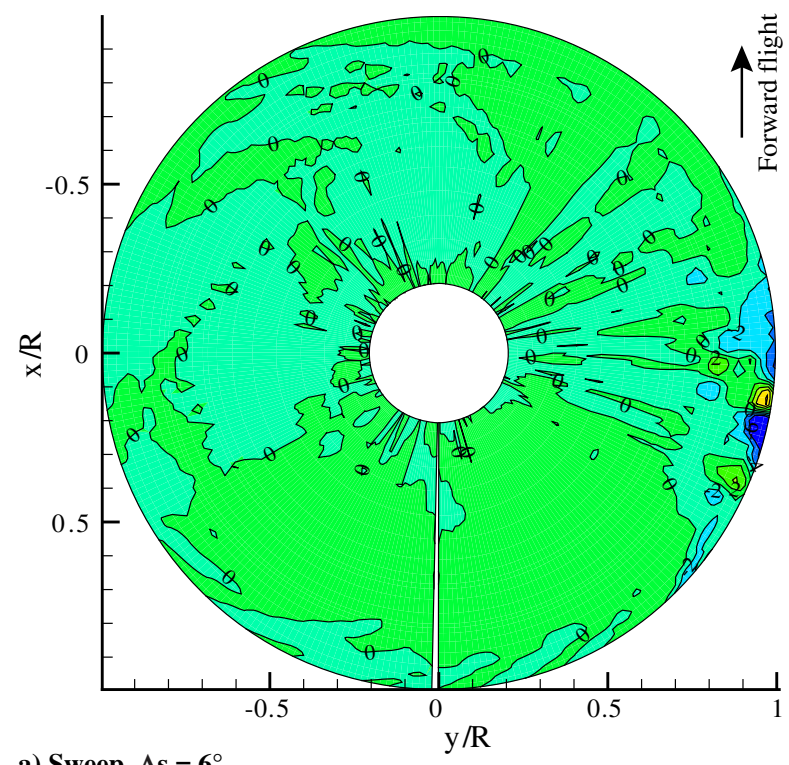

a) Sweep, $\Lambda \mathrm{s}=6^{\circ}$

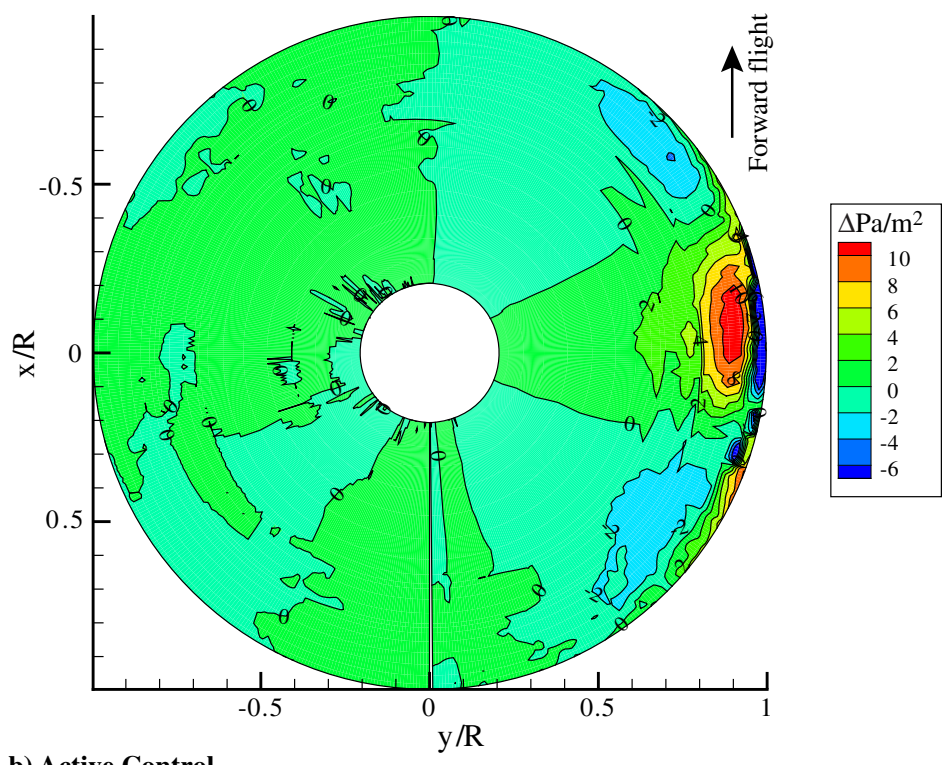

b) Active Control

Fig. 24 Change in loading noise source density due to implementation of passive/active control.

could be achieved using tip dihedral $\left(\Lambda_{a}=6 \mathrm{deg}\right)$. The insertion loss using active control was also found for a broader BPF spectrum compared to passive control. However, in all cases, there is an LFSPL increase in the out-of-plane direction. It should be noted that there is a tradeoff between in-plane LFSPL noise-reduction performance, MFSPL, and vibration performance. For OBC, in-plane LFSPL reduction below the horizon is accompanied by an increase in vibratory hub loads (Fig. 22). For tip sweep and tip anhedral, the increase in LFSPL is accompanied by a reduction in vibratory hub loads (Fig. 19). For the tip dihedral, even though LFSPL reduction is also accompanied by vibration reduction, the benefits are also offset by the increase in the MFSPL.

The loading noise plays an important role in in-plane noise reduction/amplification, even though the negative peak pressure generated by the thickness noise is the dominant contributor to the inplane noise. In the case of a swept tip blade (Fig. 18), the magnitude of total noise increased due to the in-phase reduction of the loading noise, whereas the negative peak thickness noise was reduced marginally. On the other hand, a positive peak, antiphase loading noise generated, as in the case of active control [16], would cancel the dominant negative peak thickness noise to reduce the total inplane noise.

The loading noise changes can be traced back to the rotor source using the loading noise source density before and after control. The loading noise source density is the noise due to blade loading, evaluated using Eq. (2), scaled by the local panel area at source time. For a given observer location, the loading noise source density plot shows the time and location on the rotor disk, when the noise is generated [17]. The loading source density for the baseline blade and the blade with tip sweep is shown in Fig. 23 . The two noise peaks at the observer time, $t=0.035 \mathrm{~s}$ and $t=0 . \overline{037} \mathrm{~s}$, in Figs. 18a and $18 \mathrm{~b}$ can be identifed as the two elongated regions of concentrated loading source density at $\psi \approx 90 \mathrm{deg}$.

Figure 24 shows the changes in the loading noise source density due to tip sweep $\left(\Lambda_{s}=6 \mathrm{deg}\right)$ and active control, obtained by taking the difference between the controlled and baseline case. The reduction of loading noise at the forward observer location using tip sweep (Fig. 18b) can be attributed to the reduction of the loading noise source at the tip sweep (greater than $0.9 R$ ), when the blade is at the $\psi \approx 90$ deg location (Fig. 24a). The positive loading noise [16], or antinoise, can be attributed to effects of the active flap and the blade-tip segment on the advancing side, when the blade is at the $\psi \approx 90$ deg location. By comparing Figs. 24a, 24b, it is evident that a $12 \% c$ plain flap is capable of exercising greater control authority than a $6 \mathrm{deg}, 10 \% R$ tip sweep, over the loading source contribution to counteract the thickness source noise.

\section{Conclusions}

The Active Vibration and Noise Reduction (AVINOR) code, employing a computational fluid dynamics (CFD)-based unsteady reduced-order aerodynamic model, was coupled with a noise prediction code Helicopter Noise Reduction (HELINOIR), which uses a compact loading model. Acoustic predictions from the AVINOR/HELINOIR code combination were validated against experimental results obtained in the Higher-Harmonic-Control Aeroacoustic Rotor Test (HART) and Boeing SMART rotor tests. The new code combination was also verified against blade-vortex interaction noise results obtained from the AVINOR/WOPWOP code. These comparisons indicate that the code suite produces both accurate in-plane and out-of-plane noise predictions.

The new AVINOR/HELINOIR code was employed for in-plane low-frequency sound pressure level (LFSPL) noise prediction and control at moderate advance ratios using both active and passive control methods. Passive control was implemented through three independent tip geometry modifications consisting of sweep, dihedral, and anhedral tip. A conventional plain flap using a feedback microphone located on the left boom was used for active control. The effects of both approaches on in-plane LFSPL noise and vibration control were compared. The principal conclusions of the study are summarized next.

1) The acoustic predictions from the AVINOR/HELINOIR suite were validated against experimental results from the HART I and the Boeing SMART rotor test programs. It was found that the experimental noise levels were accurately predicted. A Galerkin-type finite element model for the MBB BO105 rotor was implemented in AVINOR/HELINOIR and verified against AVINOR/WOPWOP.

2) The compact acoustic model implemented in the HELINOIR code gives accurate and computationally efficient predictions for farfield in-plane noise compared to the full pressure distribution and geometry model obtained from CFD in [25].

3) Passive control, implemented through a dihedral tip of $\Lambda_{a}=-6 \mathrm{deg}$, resulted in an in-plane noise reduction of $2 \mathrm{~dB}$ below the horizon at a moderate advance ratio $\mu=0.3$. On the other hand, the anhedral and swept tips produce LFSPL increase of up to 4 and $6 \mathrm{~dB}$, respectively, below the horizon. This shows that dihedral can be used to reduce in-plane noise. For all three tip geometry modifications, there is an insertion gain at the higher harmonics in plane and an increase in the out-of-plane LFSPL noise.

4) Tip sweep and dihedral reduce the vibratory hub loads in level flight $(\mu=0.3)$ for the cases considered. A sweep angle of $\Lambda_{s}=$ $6 \mathrm{deg}$ provides the best vibratory load reduction, reducing the $4 / \mathrm{rev}$ vertical hub shear amplitude by up to $32 \%$. The anhedral tip increases most components of the vibratory hub loads. Therefore, a 
combination of tip sweep and dihedral may be useful for in-plane LFSPL noise and vibration reduction.

5) Active closed-loop in-plane noise reduction of up to $6 \mathrm{~dB}$ was obtained below the horizon with a plain trailing-edge flap using a feedback microphone located at the tip of the left boom. However, this in-plane noise reduction was accompanied by an out-of-plane noise increase of up to $18 \mathrm{~dB}$. The insertion loss in-plane was achieved over a wider range of BPF harmonics.

6) Active in-plane LFSPL noise reduction below the horizon produces a vibration penalty. The increase in $4 / \mathrm{rev}$ vertical hub shear amplitude was approximately $60 \%$ during in-plane noise reduction using the single plain flap. There is a tradeoff between in-plane LFSPL noise reduction below the horizon, the MFSPL, and vibration performance.

7) An active plain flap, having a span of $12 \% R$ and a chord of $20 \% c$, had better control authority over loading noise source compared to a $6 \mathrm{deg}$ sweep/anhedral/dihedral of $10 \% R$ tip span considered in this study. The active flap also produced a higher penalty in the out-of-plane noise and vibratory hub loads. For the active flap, the tradeoff between hub loads, in-plane, out-of-plane noise can be controlled by varying the amplitude and phase of the flap deflection. However, passive methods do not possess such flexibility.

8) The thickness noise component is the principal contributor to in-plane noise, and it is not changed significantly by the passive or active control methods studied. Controlling the loading noise component is the primary factor for in-plane LFSPL noise reduction.

\section{Acknowledgments}

This work was supported in part by the Francois-Xavier Bagnoud Center for Rotary and Fixed Wing Air Vehicle Design (FXB-CRFWAD).

\section{References}

[1] Landgrebe, A. J., and Bellinger, E., "Experimental Investigation of Model Variable-Geometry and Ogee-Tip Rotors," NASA CR-2275, Feb. 1974.

[2] Yu, Y. H., "Rotor Blade-Vortex Interaction Noise," Progress in Aerospace Sciences, Vol. 36, No. 2, Feb. 2000, pp. 97-115. doi:10.1016/S0376-0421(99)00012-3

[3] Conner, D. A., and Hoad, D. R., "Reduction of High-Speed Impulsive Noise by Blade Planform Modification of a Model Helicopter Rotor," NASA TM84553, 1982.

[4] Baeder, J., "Passive Design for Reduction of High-Speed Impulsive Rotor Noise," Journal of the American Helicopter Society, Vol. 43, No. 3, July 1998, pp. 222-234. doi:10.4050/JAHS.43.222

[5] Murashige, A., Kobiki, N., Tsuchihasi, A., Nakamura, H., Inagaki, K., and Yamakawa, E., "ATIC Aeroacoustic Model Rotor Test at DNW," AHS International Meeting on Advanced Rotorcraft Technology and Disaster Relief, Japan Helicopter Soc., Gifu, Japan, 1998.

[6] Lyrintzis, A., Jameson, J., and Koutsavdis, E., “Technical Note: A Study of Rotorcraft Blade-Tip Shape High Speed Impulsive Noise Characteristics," Journal of the American Helicopter Society, Vol. 45, No. 1, Jan. 2000, pp. 54-57. doi:10.4050/JAHS.45.54

[7] Prieur, J., Lafon, P., Caplot, M., and Desopper, A., "Aerodynamics and Acoustics of Rectangular and Swept Rotor Blade Tips," Journal of the American Helicopter Society, Vol. 34, No. 1, Jan. 1989, pp. 42-51. doi:10.4050/JAHS.34.42

[8] Brocklehurst, A., and Barakos, G., "A Review of Helicopter Rotor Blade Tip Shapes," Progress in Aerospace Sciences, Vol. 56, Jan. 2013, pp. 35-74.

doi:10.1016/j.paerosci.2012.06.003

[9] Nishimura, H., Kondo, N., Nakamura, H., Tsujiuchi, T., Yamakawa, E., Aoyama, T., and Saito, S., "Comparison Between Calculated Rotor Noise and Experimental Data Obtained by DNW Test," Proceedings of the 24th European Rotorcraft Forum, Associazione Italiana Di Aeronautica Ed Astronautica, Marseille, France, Sept. 1998.

[10] Nakamura, H., Nishimura, H., Kondo, N., Yamakawa, E., Aoyama, T., and Saito, S., "Effect of Blade Geometry on BVI Noise in Various Flight Conditions," AHS International Meeting on Advanced Rotorcraft Technology and Disaster Relief, Japan Helicopter Soc., Gifu, Japan, 1998.
[11] Celi, R., and Friedmann, P., "Aeroelastic Modeling of Swept Tip Rotor Blades Using Finite Elements," Journal of the American Helicopter Society, Vol. 33, No. 2, April 1988, pp. 43-52. doi:10.4050/JAHS.33.43

[12] Celi, R., and Friedmann, P., "Rotor Blade Aeroelasticity in Forward Flight with an Implicit Aerodynamic Formulation," AIAA Journal, Vol. 26, No. 12, Dec. 1988, pp. 1425-1433. doi: $10.2514 / 3.10060$

[13] Friedmann, P. P., "On-Blade Control of Rotor Vibration, Noise and Vibration: Just Around the Corner? The 33rd Alexander Nikolsky Honorary Lecture," Journal of American Helicopter Society, Vol. 59, No. 4, Oct. 2014, pp. 1-37. doi:10.4050/JAHS.59.041001

[14] Sim, B. W., Janaki Ram, R. D., and Lau, B. H., "Reduced In-Plane, Low-Frequency Noise of an Active Flap Rotor," Journal of the American Helicopter Society, Vol. 59, No. 2, April 2014, pp. 1-17. doi:10.4050/JAHS.59.022002

[15] Glaz, B., Friedmann, P. P., Liu, L., Kumar, D., and Cesnik, C. E. S., "The AVINOR Aeroelastic Simulation Code and Its Application to Reduced Vibration Composite Rotor Blade Design," 50th AIAA/ASME/ASCE/ AHS/ACS Structures, Structural Dynamics and Materials Conference, AIAA Paper 2009-2601, May 2009.

[16] Chia, M., Padthe, A., Duraisamy, K., and Friedmann, P., "An Efficient Approach for the Simulation and On-Blade Control of Noise and Vibration," Proceedings of the 72nd American Helicopter Society Annual Forum, West Palm Beach, FL, May 2016.

[17] Kim, H. W., Duraisamy, K., and Brown, R. E., "Effect of Rotor Stiffness and Lift Offset on the Aeroacoustics of a Coaxial Rotor in Level Flight," Proceedings of the 65th American Helicopter Society Annual Forum, Ft Worth, TX, May 2009.

[18] Kim, H., Kenyon, A., Brown, R., and Duraisamy, K., "Interactional Aerodynamics and Acoustics of a Hingeless Coaxial Helicopter with an Auxiliary Propeller in Forward Flight," Aeronautical Journal, Vol. 113, No. 1140, Feb. 2009, pp. 65-78. doi:10.1017/S0001924000002797

[19] Ffowcs Williams, J. E., and Hawkings, D. L., "Sound Generation by Turbulence and Surfaces in Arbitrary Motion," Philosophical Transactions of the Royal Society of London, Series A, Vol. 264, No. 1151, May 1969, pp. 321-342. doi:10.1098/rsta.1969.0031

[20] Farassat, F., and Succi, G. P., "A Review of Propeller Discrete Frequency Noise Prediction Technology with Emphasis on Two Current Methods for Time Domain Calculations," Journal of Sound and Vibration, Vol. 71, No. 3, 1980, pp. 399-419. doi:10.1016/0022-460X(80)90422-8

[21] Farassat, F., and Succi, G., "The Prediction of Helicopter Rotor Discrete Frequency Noise," Vertica, Vol. 7, No. 4, Oct. 1983, pp. 309-320.

[22] Schmitz, F. H., "Rotor Noise, Aeroacoustics of Flight Vehicles, Vol. 1: Noise Sources," NASA TR 90-3052, Aug. 1991.

[23] Kelly, M. E., Duraisamy, K., and Brown, R. E., "Predicting Blade Vortex Interaction, Airloads and Acoustics Using the Vorticity Transport Model," Proceedings of the AHS Specialist's Conference on Aeromechanics, San Francisco, CA, Jan. 2008.

[24] Thom, A., and Duraisamy, K., "High Resolution Simulations of Parallel Blade-Vortex Interactions," AIAA Journal, Vol. 48, No. 10, Oct. 2010, pp. 2313-2324. doi:10.2514/1.J050381

[25] Hahn, S., Alonso, J., Duraisamy, K., Iaccarino, G., Lele, S., Moin, P., Schmitz, F., Shoeybi, M., and Wu, Z., "Progress on Hybrid Unsteady Simulation of Helicopter Rotor Flow," Annual Research Briefs, Center for Turbulence Research, Stanford Univ., Stanford, CA, 2005, pp. 121-138.

[26] Lorber, P., "Aerodynamic Results of Pressure-Instrumented Model Rotor Test at the DNW," Journal of American Helicopter Society, Vol. 36, No. 4, 1991, pp. 66-76. doi:10.4050/JAHS.36.66

[27] Padthe, A., and Friedmann, P. P., "Simultaneous Blade-Vortex Interaction Noise and Vibration Reduction in Rotorcraft Using Microflaps, Including the Effect of Actuator Saturation," Journal of American Helicopter Society, Vol. 60, No. 4, Oct. 2015, pp. 1-16. doi:10.4050/JAHS.60.042002

[28] Liu, L., Padthe, A. K., and Friedmann, P. P., "Computational Study of Microflaps with Application to Vibration Reduction in Helicopter Rotors," AIAA Journal, Vol. 49, No. 7, July 2011, pp. 1450-1465. doi:10.2514/1.J050829

[29] Yuan, K. A., and Friedmann, P., "Structural Optimization for Vibratory Loads Reduction of Composite Helicopter Rotor Blades with Advanced Geometry Tips," Journal of the American Helicopter Society, Vol. 43, 
No. 3, July 1998 , pp. 246-256. doi: $10.4050 /$ JAHS.43.246

[30] Yuan, K. A., and Friedmann, P. P., "Aeroelastic and Structural Optimization of Composite Helicopter Rotor Blades with Swept Tips," NASA CR 4665, June 1995.

[31] Millott, T. A., and Friedmann, P. P., "Vibration Reduction in Helicopter Rotors Using an Actively Controlled Partial Span Trailing Edge Flap Located on the Blade," NASA CR 4611, June 1994.

[32] Liu, L., Padthe, A., Friedmann, P. P., Quon, E., and Smith, M., "Unsteady Aerodynamics of an Airfoil/Flap Combination on a Helicopter Rotor Using CFD and Approximate Methods," Journal of American Helicopter Society, Vol. 56, No. 3, July 2011, pp. 1-13. doi:10.4050/JAHS.56.032003

[33] Patt, D., Liu, L., and Friedmann, P. P., "Simultaneous Vibration and Noise Reduction in Rotorcraft Using Aeroelastic Simulation," Journal of the American Helicopter Society, Vol. 51, No. 2, April 2006, pp. 127-140. doi:10.4050/JAHS.51.127

[34] Petot, D., "Differential Equation Modeling of Dynamic Stall," La Recherche Aérospatiale, Vol. 5, Jan. 1989, pp. 59-71.

[35] Patt, D., Liu, L., Chandrasekar, J., Bernstein, D. S., and Friedmann, P. P., "Higher-Harmonic-Control Algorithm for Helicopter Vibration Reduction Revisited," Journal of Guidance, Control, and Dynamics, Vol. 28, No. 5, Sept.-Oct. 2005, pp. 918-930. doi: $10.2514 / 1.9345$

[36] Splettstoesser, W., Seelhorst, U., Wagner, W., Boutier, A., Micheli, F., Mercker, E., and Pengel, K., "Higher Harmonic Control Aeroacoustic Rotor Test (HART) — Test Documentation and Representative Results,"
DLR, German Aerospace Center, Rept. IB 129-95/28, Cologne, Germany, Dec. 1995.

[37] Straub, F. K., Anand, V. R., Birchette, T. S., and Lau, B. H., "SMART Rotor Development and Wind Tunnel Test," Proceedings of the 35th European Rotorcraft Forum, Deutsche Gesellschaft Fuer Luft Und Raumfahrt, Hamburg, Germany, Sept. 2009.

[38] Straub, F. K., Anand, V. R., Birchette, T. S., and Lau, B. H., "Wind Tunnel Test of the SMART Active Flap Rotor," Proceedings of the 65th American Helicopter Society Annual Forum, Ft Worth, TX, May 2009.

[39] Liu, L., "BVI Induced Vibration and Noise Alleviation by Active and Passive Approaches," Ph.D. Thesis, Univ. of Michigan, Ann Arbor, 2005.

[40] Padthe, A. K., Friedmann, P. P., Chia, M. H., and Liu, L., "A Comprehensive Numerical Assessment of Microflaps for On-Blade Control of Rotorcraft Noise and Vibration," Journal of Aircraft, Vol. 53, No. 4, Oct. 2016, pp. 1113-1130. doi:10.2514/1.C033448

[41] de Terlizzi, M., "Blade Vortex Interaction and Its Alleviation Using Passive and Active Control Approaches," Ph.D. Thesis, University of California, Los Angeles, Los Angeles, CA, 1999.

[42] Wilke, G., "Applying Multi-Objective Variable-Fidelity Optimization Techniques to Industrial Scale Rotors: Blade Designs for Clean Sky," Proceedings of the 41st European Rotorcraft Forum, Deutsche Gesellschaft Fuer Luft Und Raumfahrt, Munich, Germany, Sept. 2015.

[43] Patt, D., Liu, L., and Friedmann, P. P., "Rotorcraft Vibration Reduction and Noise Prediction Using a Unified Aeroelastic Response Simulation," Journal of the American Helicopter Society, Vol. 50, No. 1, Jan. 2005, pp. 95-106. doi: $10.4050 / 1.3092846$ 